\title{
On Quinn's Invariants of 2-dimensional CW-complexes
}

\author{
Ivelina Bobtcheva
}

\begin{abstract}
Given a semisimple stable autonomous tensor category $\mathcal{A}$ over a field $K$, to any group presentation with finite number of generators we associate an element $Q(P) \in K$ invariant under the Andrews-Curtis moves. We show that in fact, this is the same invariant as the one produced by the algorithm introduced by Frank Quinn in [8]. The new definition allows us to present a relatively simple proof of the invariance and to evaluate $Q(P)$ for some presentations. On the basis of some numerical calculations over different Gelfand-Kazhdan categories, we make a conjecture which allows us to relate the value of $Q(P)$ for two different classes of presentations.
\end{abstract}

\section{Introduction}

The original formulation of the Andrews-Curtis conjecture [i] has the following algebraic form. Suppose that $<x_{1}, x_{2}, \ldots, x_{n} \mid R_{1}, R_{2}, \ldots, R_{n}>$ is a presentation of the trivial group. Then this can be reduced to the empty presentation by a finite sequence of operations of the following types:

(i) The places of $R_{1}$ and $R_{s}$ are interchanged.

(ii) $R_{1}$ is replaced with $g R_{1} g^{-1}$, where $g$ is any element in the group.

(iii) $R_{1}$ is replaced with $R_{1}^{-1}$.

(iv) $R_{1}$ is replaced with $R_{1} R_{2}$.

(v) Adding of an additional generator $y$ and an additional relator $y$.

(vi) The reverse of (v).

We will refer to these six operations as AC-moves. The Andrews-Curtis conjecture is a particular case of the more general deformation conjecture stating that a simple homotopy equivalence of 2-complexes can be obtained by a deformation through 2-complexes (2-deformation 1). It is known that if two complexes $X$ and $Y$ are simple homotopy equivalent, then for some $k$, there exists a 2-deformation from the one point union of $X$ with $k$ copies of $S^{2}$ to the one point union of $Y$ with $k$ copies of $S^{2}$.

An algebraic formulation of this deformation conjecture comes from the following result [10]: two 2-complexes are simple homotopy equivalent if and only

1991 Mathematics Subject Classification. Primary 57M20; Secondary 57M05.

Partially supported by grant from the US National Science Fondation.

${ }^{1}$ In part of the literature [5] this is actually called a 3-deformation, since it can be achieved through expansions and collapses of disks of dimension at most three.

(C)0000 (copyright holder) 
if the corresponding group presentations have the same difference \# relation -\# generators, and they can be reduced to the same group presentation via the moves (i)-(vi) above plus the additional move

(vii) $<x_{1}, x_{2}, \ldots, x_{n} \mid R_{1}, R_{2}, \ldots, R_{m}>$ is replaced with $<x_{1}, x_{2}, \ldots, x_{n} \mid Q_{1}, Q_{2}, \ldots, Q_{m}>$ if there are words $w_{i, j}, v_{i, j}$ for $1 \leq i \leq m$, $1 \leq j \leq k(i)$, and indices $r(i, j), s(i, j)$ such that for all $i$,

$$
R_{i}\left(Q_{i}\right)^{-1}=\sqcap_{j=1}^{k(i)} R_{i, j} Q_{i, j} R_{i, j}^{-1} Q_{i, j}^{-1},
$$

where $R_{i, j}=w_{i, j} R_{r(i, j)}^{ \pm 1} w_{i, j}^{-1}$ and $Q_{i, j}=v_{i, j} Q_{s(i, j)}^{ \pm 1} v_{i, j}^{-1}$. Then, in its general form, the Andrews-Curtis conjecture states that two presentations which have the same difference \# relation - \# generators and are equivalent through the moves (i)-(vii), are actually equivalent through the moves (i)-(vi).

In [8] an algorithm is described for computing topological quantum field theories on 1+1-complexes associated to stable subcategories of the autonomous tensor categories of Gelfand and Kazhdan over the finite field $Z_{p}$. These two dimensional topological theories have the property that the invariant corresponding to $S^{2}$ is 0 , and therefore give the opportunity of looking for counterexamples of the AndrewsCurtis conjecture. The invariants have been approached numerically 8 , 2, 9 where every CW-complex is being sliced in standard elementary slices. Then the invariant of the complex is obtained by composing the morphisms corresponding to each slice. Up to now no counterexamples have been detected, and the analytical value of the invariant even for simple complexes wasn't known.

The goal of this work is to present a "global" definition of the invariant, in sense that, given a group presentation $P$ with a finite number of generators, we associate to it an element $Q(P) \in K$, which is invariant under the AC-moves. There is an explicit formula for $Q(P)$ in terms of some kind of trace (partial trace ) of a given morphism in the underlying semisimple stable autonomous tensor category $\mathcal{A}$.

The paper is structured in the following way. In section 2 some basic definitions and properties of semisimple stable autonomous tensor categories are presented. In section 3 the diagrammatic notation, which will be the main tool of manipulating the expressions is described. Section 4 is concerned with the definition and properties of partial traces in the category. The invariant $Q(P)$ is defined in section 5 , and the proof of the invariance under the AC-moves is presented in section 6 . Section 7 reviews the algorithm introduced in [8] and shows that it produces the same invariant. In the last section we list some conjectures on the dimension functions in the Gelfand-Kazhdan categories and on the basis of one of them show that if $P^{\prime}$ is a presentation obtained from $P$ by adding a generator $y$ and a relator $x y x^{-1} y^{-1}$ (where $x$ is a generator of $P$ ), and if $P^{\prime \prime}$ is obtained from $P$ by simply adding a relator $x$, then $Q\left(P^{\prime}\right)=N Q\left(P^{\prime \prime}\right)$, where $N$ is the number of the simple objects in the category.

I would like to thank Frank Quinn for introducing me into the subject, and patiently explaining to me many basic concepts and ideas. His help and encouragement have been decisive for the completion of this work.

\section{General category statements}


2.1. $\mathcal{A}$ is a tensor category if it is supplied with a bifunctor $\diamond: \mathcal{A} \times \mathcal{A} \rightarrow \mathcal{A}$ and an identity object $\mathbf{1}$, such that for any $A, B, C \in \mathcal{A}$ there are natural isomorphisms

$$
\begin{aligned}
& \alpha_{A, B, C}:(A \diamond B) \diamond C \rightarrow A \diamond(B \diamond C) \quad \text { (associativity morphisms), } \\
& \eta_{A}: \mathbf{1} \diamond A \rightarrow A, \quad{ }_{A} \eta: A \diamond \mathbf{1} \rightarrow A, \\
& \gamma=\gamma_{A, B}: A \diamond B \rightarrow B \diamond A \quad \text { (commutativity morphisms). }
\end{aligned}
$$

Moreover, $\gamma_{A B} \gamma_{B A}=i d_{A B}$. These isomorphisms satisfy a number of axioms as listed in [7].

In order to simplify the notation, in the future we will often write $A B$ instead of $A \diamond B$.

2.2. Let $\mathcal{P}$ be the free $(\otimes, I)$-algebra on a single symbol $O$. The number of $O$ 's in an element $T$ in $\mathcal{P}$ will be called its length. Let now $\mathcal{A}$ be a tensor category,. Following [6], we define a category $\mathcal{P} \circ \mathcal{A}$, with objects $T[\underline{B}] \equiv T\left[B_{1}, B_{2}, \ldots, B_{n}\right]$, where $T$ is an object of length $n$ in $\mathcal{P}$, and $\underline{B}=\left(B_{1}, B_{2}, \ldots, B_{n}\right)$ is a sequence of objects in $\mathcal{A}$. The morphisms have the form $\pi\left[f_{1}, f_{2}, \ldots, f_{n}\right]: T\left[B_{1}, B_{2}, \ldots, B_{n}\right] \rightarrow$ $S\left[A_{1}, A_{2}, \ldots, A_{n}\right]$, where $\pi$ is a bijection from the $O$ 's in $T$ to the $O$ 's in $S$, i.e. an element in the symmetric group $\mathbf{S}_{n}$, and $f_{i}: B_{\pi^{-1}(i)} \rightarrow A_{i}$ are maps in $\mathcal{A}$. We use the following convention for the special maps:

$$
\begin{aligned}
& T\left[f_{1}, f_{2}, \ldots, f_{n}\right] \equiv \iota(n)\left[f_{1}, f_{2}, \ldots, f_{n}\right]: T\left[B_{1}, B_{2}, \ldots, B_{n}\right] \rightarrow T\left[A_{1}, A_{2}, \ldots, A_{n}\right], \\
& \pi \equiv \pi\left[i d_{A_{1}}, i d_{A_{2}}, \ldots, i d_{A_{n}}\right]: T\left[A_{\pi(1)}, A_{\pi(2)}, \ldots, A_{\pi(n)}\right] \rightarrow S\left[A_{1}, A_{2}, \ldots, A_{n}\right],
\end{aligned}
$$

where $f_{i}: B_{i} \rightarrow A_{i}$, and $\iota(n)$ denotes the identity element $\mathbf{S}_{\mathbf{n}}$. We will refer to the objects in $\mathcal{P} \circ \mathcal{A}$ as bracketings in $\mathcal{A}$, and to the maps of type $\pi=$ $\pi\left[i d_{A_{1}}, i d_{A_{2}}, \ldots, i d_{A_{n}}\right]$ as permutations.

2.3. $\mathcal{P} \circ \mathcal{A}$ possesses a structure of a tensor category with identity object $I[]$, and product $\otimes$ defined in the following way:

$$
S\left[B_{1}, B_{2}, \ldots, B_{n}\right] \otimes T\left[B_{n+1}, B_{2}, \ldots, B_{m}\right]=(S \otimes T)\left(B_{1}, B_{2}, \ldots, B_{n+m}\right) .
$$

The associativity and commutativity morphisms are defined to be the corresponding permutations. Then the coherence theorem of MacLane [7] is equivalent to the assertion that there is a strict tensor functor $\mathcal{P} \circ \mathcal{A} \rightarrow \mathcal{A}$. We write $T\left(B_{1}, B_{2}, \ldots, B_{n}\right)$ and $\pi\left(f_{1}, f_{2}, \ldots, f_{n}\right)$ for the images of $T\left[B_{1}, B_{2}, \ldots, B_{n}\right]$ and $\pi\left[f_{1}, f_{2}, \ldots, f_{n}\right]$ under this functor; for example $((O \otimes O) \otimes I)(a, b)=(a \diamond b) \diamond \mathbf{1}$. The images of the permutations will be called again permutations, and we will denote them with the corresponding cyclic decomposition. For example, $(1,2)(3,5,4): T(A, B, C, D, G) \rightarrow$ $S(B, A, D, G, C)$.

In the case when $B_{i}=B$ for any $i$, we will use the convention $T\left(B^{k}\right)=$ $T\left(B_{1}, B_{2}, \ldots, B_{k}\right)$. Then the permutations define an action of $\mathbf{S}_{k}$ on $T\left(B^{k}\right)$, and we denote the corresponding representation of $\mathbf{S}_{k}$ as $\rho\left[T\left(B^{k}\right)\right]$.

2.4. Let $P \mathcal{A}$ be the category with the same objects as $\mathcal{P} \circ \mathcal{A}$, but has different set of morphisms: for any morphism $T(\underline{B}) \rightarrow R(\underline{B})$ in $\mathcal{A}$ there is a morphism $T[\underline{B}] \rightarrow R[\underline{C}]$, which will be denoted in the same way. The composition is obvious. Then there are functors $\mathcal{P} \circ \mathcal{A} \rightarrow P \mathcal{A}$ and $P \mathcal{A} \rightarrow \mathcal{A}$, whose compositions is exactly the functor $\mathcal{P} \circ \mathcal{A} \rightarrow \mathcal{A}$ given by the coherence result of MacLane. 
2.5. An autonomous tensor category in the terminology of [11 is a tensor category $\mathcal{A}$ in which to every object $A$ is assigned an object $A^{*} \in \mathcal{A}$ and morphisms $\Lambda_{A}: \mathbf{1} \rightarrow A^{*} A$ (coform) and $\lambda_{A}: A A^{*} \rightarrow \mathbf{1}$ (form) such that the compositions

$$
\begin{aligned}
& A^{*} \stackrel{\eta_{A^{*}}-1}{\longrightarrow} 1 A^{*} \stackrel{\Lambda_{A} \diamond i d_{A^{*}}}{\longrightarrow}\left(A^{*} A\right) A^{*} \stackrel{\alpha}{\longrightarrow} A^{*}\left(A A^{*}\right) \stackrel{i d_{A^{*} \diamond \lambda_{A}}}{\longrightarrow} A^{*} \mathbf{1} \stackrel{\eta_{A^{*}}}{\longrightarrow} A^{*}, \\
& A^{A} \stackrel{\eta^{-1}}{\longrightarrow} A 1 \stackrel{i d_{A} \diamond \Lambda_{A}}{\longrightarrow} A\left(A^{*} A\right) \stackrel{\alpha^{-1}}{\longrightarrow}\left(A A^{*}\right) A^{\lambda_{A} \diamond i d_{A}} \longrightarrow 1 A \stackrel{\eta_{A}}{\longrightarrow} A
\end{aligned}
$$

act as identities. In any such category there is a canonical isomorphism $w_{A}: A^{* *} \rightarrow$ $A$, for which $\gamma_{A^{*} A} \circ \Lambda_{A}=\left(w_{A} \diamond i d\right) \circ \Lambda_{A^{*}}$ We call an autonomous tensor category $\mathcal{A}$ stable if $\left(A^{*}\right)^{*}=A$ and $w_{A}=i d_{A}$.

2.6. A category $\mathcal{A}$ is called semisimple if it is abelian over a field $K$, and if there is a finite subset of objects $\Sigma$ in $\mathcal{A}$, such that every other objects is isomorphic to a direct sum of objects in $\Sigma$, and for any $a, b \in \Sigma, h_{\mathcal{A}}(a, b) \simeq\left\{\begin{array}{l}0, \text { if } a \neq b, \\ K, \text { if } a=b .\end{array}\right.$. In general, we will use small latin letters $a, b, c, \ldots$ to denote objects in $\Sigma$, and capital letters to indicate an arbitrary object in the category. We also use the notation $F(A, B)=\operatorname{hom}_{\mathcal{A}}(A, B)$.

The fact that an object $A$ is isomorphic to a direct sum of objects in $\Sigma$ means that for any $a \in \Sigma$ there exists a basis $\left\{\epsilon_{i}(a, A): a \rightarrow A\right\}_{i}$ of $F(a, A)$ and a basis $\left\{\epsilon_{i}(a, A)^{*}: A \rightarrow a\right\}_{i}$ of $F(A, a)$, which are dual in sense that $\epsilon_{i}(a, A)^{*} \circ \epsilon_{j}(a, A)=$ $\delta_{i, j} i d_{a}$. Moreover, $\sum_{a, j} \epsilon_{j}(a, A) \circ \epsilon_{j}(a, A)^{*}=i d_{A}$. Then, given the objects $A, B$ and $C$, the map

$$
\begin{array}{cl}
\nabla: \oplus_{b \in \Sigma} F(a, A \diamond(b \diamond C)) \otimes F(b, B) \rightarrow & F(a, A \diamond(B \diamond C)), \\
\psi \otimes \phi \rightarrow & \left(i d_{A} \diamond\left(\phi \diamond i d_{C}\right)\right) \circ \psi
\end{array}
$$

is an isomorphism. In fact, the inverse of $\nabla$ is given by the map

$$
\begin{array}{cl}
\nabla^{-1}: F(a, A \diamond(B \diamond C)) \rightarrow & \oplus_{b \in \Sigma} F(a, A \diamond(b \diamond C)) \otimes F(b, B), \\
\phi \rightarrow & \oplus_{b \in \Sigma, i}\left[\left(i d_{A} \diamond\left(\epsilon_{i}(b, B)^{*} \diamond i d_{C}\right)\right) \circ \phi\right] \otimes \epsilon_{i}(b, B) .
\end{array}
$$

In a semisimple tensor category for any simple object $b$, the representation $\rho\left[T\left(a^{k}\right)\right]$ from 2.3, induces representations $\rho_{b}\left[T\left(a^{k}\right)\right]$ of $\mathbf{S}_{\mathbf{k}}$ on the space $F\left(b, T\left(a^{k}\right)\right)$.

2.7. The concrete categories, used in the numerical computations of quantum invariants of 2-dimensional CW-complexes, are the ones studied in 4 . These are semisimple tensor categories, defined over the finite field $K=Z_{p}$, and are constructed as a quotient of the category of some modular finite-dimensional modules of a semisimple algebraic group $G$, in the case when $p$ is greater then the Coxeter number of the corresponding Lie-algebra. If $\mathcal{A}$ is such Gelfand-Kazhdan category, its simple objects correspond to the simple modules with highest weights inside the fundamental alcove. We remind that involution on a set $S$ is a bijection of $S$ into itself whose square is equal to the identity. Then on the set of simple objects $\Sigma$ there is an involution denoted by $a \rightarrow a^{*}$, such that $a^{*}$ is isomorphic to the dual module of $a$. A form and coform can be introduced, and this makes $\mathcal{A}$ an autonomous tensor category. But this category is not always stable, i.e. the condition $\gamma_{a, a^{*}} \circ \Lambda_{a^{*}}=\Lambda_{a}: \mathbf{1} \rightarrow a^{*} \diamond a$. is not always satisfied. The simplest examples come from the categories of modular representations of $\operatorname{sl}(2)$. In this case if $a$ is an odd highest weight module in $\Sigma, \gamma_{a, a^{*}} \circ \Lambda_{a^{*}}=-\Lambda_{a}$. But the subcategory generated by the even highest weights modules is stable. 
The general situation is not very different from the one of $s l(2)$, and is described, for example, in [2. It is shown that there is always a subcategory of $\mathcal{A}$ which is stable, for example the one generated by the modules with highest weights in the root lattice. In general, there are number of sublattices of the weight lattice which produce subcategories with the desired property.

\section{Diagrammatic notation}

The diagrammatic notation used here is based on the coherence results in 6, 11, 3. The particular case of autonomous tensor categories is studied in $[\mathbf{6}]$, and the results there are extended to tortile categories in [11, 3]. The version we use here is the one described in [12, specified to the case of stable autonomous tensor category.

The idea behind the diagrammatic notation is that to each composition of $\mathcal{A}$ morphisms there corresponds a labeled link diagram with coupons. Then there are rules for transforming these diagrams, and the statement is that if by such transformations the diagrams corresponding to $\psi_{1}: A \rightarrow B$ and $\psi_{2}: A \rightarrow B$ can be reduced to the same diagram, then $\psi_{1}=\psi_{2}$. In this sense, a morphism in $\mathcal{A}$ is determined by its diagram.

Proposition 3.1. Suppose that the group $Z_{2} \times Z_{2}=<\theta_{1}, \theta_{-1} \mid \theta_{1}^{2}, \theta_{-1}^{2}>$ acts on a set $S$. Let $S_{i}, i= \pm 1$, be the set of fixed points of the subgroup generated by $\theta_{i}$, and suppose that $S_{1} \cap S_{-1}=\emptyset$. Then if $\tilde{S}=S \backslash\left(S_{1} \cup S_{-1}\right)$ and $\mathcal{O}$ is any orbit, we have that either $\mathcal{O} \backslash \tilde{S}$ is a two elements set, or $\mathcal{O} \subset \tilde{S}$.

The proof is straightforward.

3.2. The coupon category $\mathbf{C} \mathcal{A}$ associated to a stable autonomous tensor category $(\mathcal{A}, \alpha, \gamma, \eta, \lambda, \Lambda, *)$ is defined as a quotient of another category $\tilde{\mathbf{C A}}$. The objects of $\tilde{\mathbf{C A}}$ are finite sequences of objects in $\mathcal{A}$. Below, we will use the notation $\underline{A}$ both for the sequence $\left(A_{1}, A_{2}, \ldots, A_{l}\right)$ of length $|\underline{A}|=l$, and for the set of objects $\left\{A_{i}\right\}_{i=1}^{l}$ in $\mathcal{A}$. In particular, $\underline{A} \sqcup \underline{B}$ denotes the disjoint union of the two sets, and $\underline{A} \backslash \underline{B}$ denotes the complement of $\underline{B}$ in $\underline{A}$.

We introduce a $*$ operation on the objects in $\tilde{\mathbf{C} \mathcal{A}:} \underline{A}=\left(A_{1}, A_{2}, \ldots, A_{l}\right) \rightarrow$ $\underline{A}^{*}=\left(A_{l}^{*}, A_{l-1}^{*}, \ldots, A_{1}^{*}\right)$. Then a morphism $(F, \theta): \underline{X} \rightarrow \underline{Y}$ in $\tilde{\mathbf{C A}}$ is given by a finite set $F=\left\{f^{i}: T^{i}\left[\underline{A}^{i}\right] \rightarrow R^{i}\left[\underline{B}^{i}\right]\right\}_{i}$ of morphisms in $P \mathcal{A}$, and an involution $\theta$ on the set $S_{F}=\underline{X} \sqcup \underline{Y}^{*} \sqcup\left(\sqcup_{i}\left(\underline{A}^{i *} \sqcup \underline{B}^{i}\right)\right)$ such that $\theta(C)^{*}=C$ and $\theta$ is free of fixed points. The elements of $\underline{A}^{i}$ and $\underline{B}^{i}$ are called correspondingly the lower and the upper ends of $f^{i}$. The identity morphisms in $\tilde{\mathbf{C A}}, i d: S \rightarrow S$ are given by $(\{\}, *)$.

Let now $(F, \theta): \underline{X} \rightarrow \underline{Y}$ and $(G, \eta): \underline{Y} \rightarrow \underline{Z}$ be two morphisms in $\tilde{\mathbf{C A}}$. To define their composition, we would like to take $F \sqcup G$ with the involution map obtained by identifying the two copies of $\underline{Y}$, but in this way one obtains an involution, which may contain fixed points. To avoid this, identity morphisms are introduced as follows.

Let $S^{\prime}=S_{F} \backslash \underline{Y}^{*}$ and $S^{\prime \prime}=S_{G}^{*} \backslash \underline{Y}^{*}$. We extend $\theta$ to an involution on $S=S^{\prime} \sqcup \underline{Y}^{*} \sqcup S^{\prime \prime}$ by defining $\theta$ to be the identity on the elements in $S^{\prime \prime}$. In a similar way we define an involution $\eta^{\prime}$ on $S$ as $\eta^{\prime}(x)=\left\{\begin{array}{c}\eta\left(x^{*}\right)^{*} \text { if } x \in \underline{Y}^{*} \sqcup S^{\prime \prime} \\ x \text { otherwise }\end{array}\right.$. Then $\theta$ and $\eta^{\prime}$ define an action of $Z_{2} \times Z_{2}$ on $S$, which satisfy the requirements of proposition 3.1, where $\tilde{S}=\underline{Y}^{*}, S_{1}=S^{\prime}$, and $S_{-1}=S^{\prime \prime}$. Then, according to the proposition, the orbits under this action are divided in two - the ones which are 
entirely contained in $\underline{Y}^{*}$, and ones which have exactly two elements outside this set. Let $\mathbf{E}$ be the subset of orbits which are entirely contained in $\underline{Y}^{*}$. If $\mathcal{O} \in \mathbf{E}$, let $A_{\mathcal{O}}$ be the smallest element in the orbit ( with respect to the ordering which comes with $\left.\underline{Y}^{*}\right)$, and let $\mathbf{T}=\left\{i d_{A_{\mathcal{O}}}\right\}_{\mathcal{O} \in \mathbf{E}}$. Then composition of $(F, \theta)$ and $(G, \eta)$ is given by $(F \sqcup G \sqcup T, \xi)$, where $\xi$ is an involution on the set $S^{\prime} \sqcup\left(S^{\prime \prime}\right)^{*} \sqcup\left(\sqcup_{\mathcal{O} \in \mathbf{E}}\left\{A_{\mathcal{O}}^{*}, A_{\mathcal{O}}\right\}\right)$ defined in the following way. Let $\imath: S^{\prime} \sqcup\left(S^{\prime \prime}\right)^{*} \stackrel{i d \sqcup *}{\longrightarrow} S^{\prime} \sqcup S^{\prime \prime} \subset S$. Then if $A$ is an element in $S^{\prime} \sqcup\left(S^{\prime \prime}\right)^{*}$, we define $\xi(A)=\imath^{-1}(B)$, where $B$ is the unique element in $S^{\prime} \sqcup S^{\prime \prime}$ which belongs to the same $Z_{2} \times Z_{2}$-orbit as $\imath(A)$. Moreover, $\xi\left(A_{\mathcal{O}}\right)=A_{\mathcal{O}}^{*}$.

3.3. The coupon category $\mathbf{C} \mathcal{A}$ is defined to be the quotient of $\tilde{\mathbf{C A}} \mathcal{A}$ under the following relations. Let $(F, \theta)$ be a morphism in $\tilde{\mathbf{C A}} \mathcal{A}$. Then the following replacements between morphisms in $F$ can be made:

(a) $i d_{A}: O[A] \rightarrow O[A]$, whose ends are not mapped into each other, can be removed. Then $\theta$ changes to $\theta^{\prime}$ where $\theta^{\prime}(X)$ is equal to $\theta(X)$ if $X$ is not mapped into some of the ends of $i d_{A}$, otherwise $\theta^{\prime}(\theta(A))=\theta\left(A^{*}\right)$.

(b) If $f: T[\underline{A}] \rightarrow Q[\underline{B}]$ and $g: Q[\underline{C}] \rightarrow R[\underline{D}]$ are such that $|\underline{B}|=|\underline{C}|$ and the upper ends of $f$ are mapped into the lower ends of $g$, i.e. $\theta\left(B_{i}\right)=C_{i}^{*}$, then $f, g$ can be replaced with $f \circ g$.

(c) $f: T[\underline{A}] \rightarrow Q[\underline{B}] \longleftrightarrow f^{\prime}=\iota(l)^{-1} \circ f \circ \iota(k): T^{\prime}[\underline{A}] \rightarrow Q^{\prime}[\underline{B}]$, where $k=|\underline{A}|$, $l=|\underline{B}|$, and $\iota(k): T^{\prime}(\underline{A}) \rightarrow T(\underline{A})$ and $\iota(l): Q^{\prime}(\underline{B}) \rightarrow Q(\underline{B})$ are the identity permutations as defined in 2.2.

(d) $\gamma_{A, B}:(O \otimes O)[A, B] \rightarrow(O \otimes O)[B, A] \longleftrightarrow i d:(O \otimes O)[A, B] \rightarrow(O \otimes$ $O)[A, B]$.

(e) $\left\{f: T[\underline{A}] \rightarrow T^{\prime}[\underline{B}], g: Q[\underline{C}] \rightarrow Q^{\prime}[\underline{D}]\right\} \longleftrightarrow g \diamond f: T[\underline{A}] \otimes Q[\underline{B}] \rightarrow$ $T^{\prime}[\underline{C}] \otimes Q^{\prime}[\underline{D}]$.

(f) $\eta_{A}:(O \otimes I)[A] \rightarrow O[A] \longleftrightarrow i d_{A}: O[A] \rightarrow O[A]$, and the analogous statement for ${ }_{A} \eta$.

(g) $\lambda_{A}:(O \otimes O)\left[A, A^{*}\right] \rightarrow I[\{\}] \longleftrightarrow i d_{A^{*}}: O\left[A^{*}\right] \rightarrow O\left[A^{*}\right]$.

(h) $\Lambda_{A}: I[\{\}] \rightarrow(O \otimes O)\left[A^{*}, A\right] \longleftrightarrow i d_{A}: O[A] \rightarrow O[A]$.

3.4. The category $\mathbf{C} \mathcal{A}$ is a stable autonomous tensor category. The product $\underline{A} \otimes \underline{B}$ is simply the sequence obtained by putting the elements of $\underline{B}$ after the elements of $\underline{A}$, the identity object is the empty set, and the associativities, $\eta_{\underline{A}}$ and $\underline{A} \eta$ are the identity morphisms. $\gamma, \lambda$ and $\Lambda$ are given by $(\{\}, *)$. Moreover, there is a strict tensor functor $P \mathcal{A} \rightarrow \mathbf{C} \mathcal{A}$ which maps $T[\underline{B}]$ into $\underline{B}$ and $f: T[\underline{B}] \rightarrow R[\underline{C}]$ into $(\{f\}, *): \underline{B} \rightarrow \underline{C}$. Then the coherence result which underlines the use of the diagrams is that if $\psi, \phi: T(\underline{B}) \rightarrow R(\underline{C})$ are two morphisms in $\mathcal{A}$ which have the same image in $\mathbf{C} \mathcal{A}$, then $\psi=\phi$. In this way, given $T, R \in \mathcal{P}$, every morphism $(F, \theta): \underline{B} \rightarrow \underline{C}$ determines a unique morphism $T(\underline{B}) \rightarrow R(\underline{C})$ in $\mathcal{A}$, which also will be denoted as $(F, \theta)$.

3.5. A morphism $(F, \theta): \underline{X} \rightarrow \underline{Y}$ in $\mathbf{C A}$ can be described by a diagram in the following way. First we draw a set of $|\underline{X}|+|\underline{Y}|$ points labeled by the corresponding elements in $\underline{X}$ and $\underline{Y}$, and for each morphism $f: T[\underline{A}] \rightarrow R[\underline{B}]$ we draw a rectangle (coupon) labeled by $f$ with $|\underline{A}|$ lines labeled by $A_{i}$ 's, attached to its lower side, and $\underline{\mid B} \mid$ lines labeled by $B_{j}$ 's, attached to its upper side. Then we connect with lines every two ends of coupons and labeled points which are mapped into each other by the involution $\theta$. In this way one looses any information about the elements in $\mathcal{P}$ associated with the morphism of a coupon except their length, but note that 
according to 3.3 (c) this is the only essential one. An example for the type of diagram obtained in this way is presented in figure 1. Then the equivalence relations in 3.3 imply that one can perform the local diagram moves represented on figure 2. Note that if in the diagram there is a line which starts and finishes in a coupon and is labeled by the identity object $\mathbf{1}$, this line can be removed.

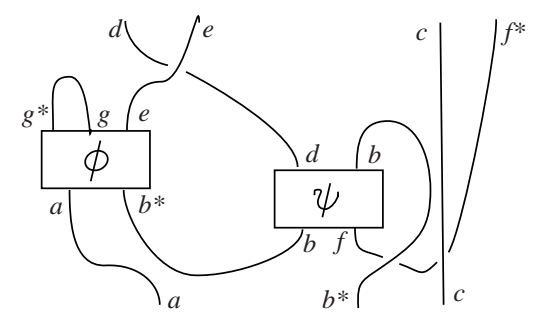

Figure 1. General diagram.

3.6. We introduce a special notation for the basic elements of the homomorphism spaces. If $\left\{\epsilon_{i}\right\}_{i}$ is a basis of $F\left(b, T\left(A_{1}, A_{2}, \ldots, A_{l}\right)\right)$ and $\left\{\epsilon_{i}^{*}\right\}_{i}$ is its dual basis of $F\left(T\left(A_{1}, A_{2}, \ldots, A_{l}\right), b\right)$ (see 2.6), we represent these basic elements in the diagrams as it is shown in figure 3 (a) and (b). In the special and most often used case of a basis for $F(a, b c)$, where $a, b, c \in \Sigma$, the notation will be simplified by replacing the coupon with a vertex labeled by $i$. Figure 1 represents the defining identities of the dual basis. From the discussion in 2.6 it follows that if $\left\{\zeta_{k}\right\}_{k}$ is a basis for $F(a, b C)$ and $\left\{\epsilon_{i}\right\}_{i}$ is a basis for $F(b, A B)$, then as one varies $b, i, k$, the morphisms $\left(\epsilon_{i} \diamond i d_{C}\right) \circ \zeta_{k}$ form a basis of $F(a,(A B) C)$ (figure 3 (c)).

3.7. Given a morphism $\varphi$ of $F(A, B)$, we define $\hat{\varphi}=\eta_{A^{*}}\left(\lambda_{B^{*}} \diamond i d_{A^{*}}\right) \alpha_{B^{*}, B, A^{*}}^{-1}\left(i d_{B^{*}} \diamond\left(\varphi \diamond i d_{A^{*}}\right)\right)\left(i d_{B^{*}} \diamond \Lambda_{A^{*}}\right)\left(B_{B^{*}} \eta\right)^{-1} \in F\left(B^{*}, A^{*}\right)$.

The corresponding diagram is presented in figure 5. It is easy to see that if $\left\{\epsilon_{i}\right\}_{i}$ is a basis for $F(A, B)$, then $\left\{\hat{\epsilon}_{i}\right\}_{i}$ is a basis for $F\left(B^{*}, A^{*}\right)$ with dual $\left\{\hat{\epsilon}_{i}^{*}\right\}_{i}$.

3.8. If $\varphi: A \rightarrow A$ is a morphism in $\mathcal{A}$, define the rank of $\varphi$ to be the morphism $r(\varphi)=\lambda_{A^{*}} \circ\left(i d_{A^{*}} \diamond \varphi\right) \circ \Lambda_{A}$, and $r(A) \equiv r\left(i d_{A}\right)$. We think of $r(\varphi)$ as an element in the field via the standard isomorphism $F(\mathbf{1}, \mathbf{1}) \sim K$. The diagrammatic notation for the rank of a morphism is presented in figure 6 (a). As an easy application of the diagrams one can see that

(a) $r(A)=r\left(A^{*}\right)$;

(b) $r\left(T\left(A_{1}, A_{2}, \ldots, A_{l}\right)\right)=r\left(A_{1}\right) r\left(A_{2}\right) \ldots r\left(A_{l}\right)$;

(a) allows as to use the simplified notation on figure 6 (b) for a rank of an object. The identity in figure 6 (c) follows from the fact that for any simple object $b$, $F(b, b) \sim K$.

Let $\left\{\epsilon_{i}(a, b c)\right\}_{i}$ be a basis for $F(a, b c)$, and $\left\{\epsilon_{i}(a, b c)^{*}\right\}_{i}$ be the corresponding dual basis. Then the morphisms $\bar{\zeta}_{i}\left(c, b^{*} a\right)=\eta_{c}\left(\lambda_{b^{*}} \diamond i d_{c}\right)\left(i d_{b^{*}} \diamond \epsilon_{i}\right)$ and $\zeta_{i}\left(c, b^{*} a\right)=$ $\left(i d_{b^{*}} \diamond \epsilon_{i}^{*}\right)\left(\Lambda_{b} \diamond i d_{c}\right) \eta_{c}{ }^{-1}$ represent sets of elements in $F\left(b^{*} a, c\right)$ and $F\left(c, b^{*} a\right)$ correspondingly. 

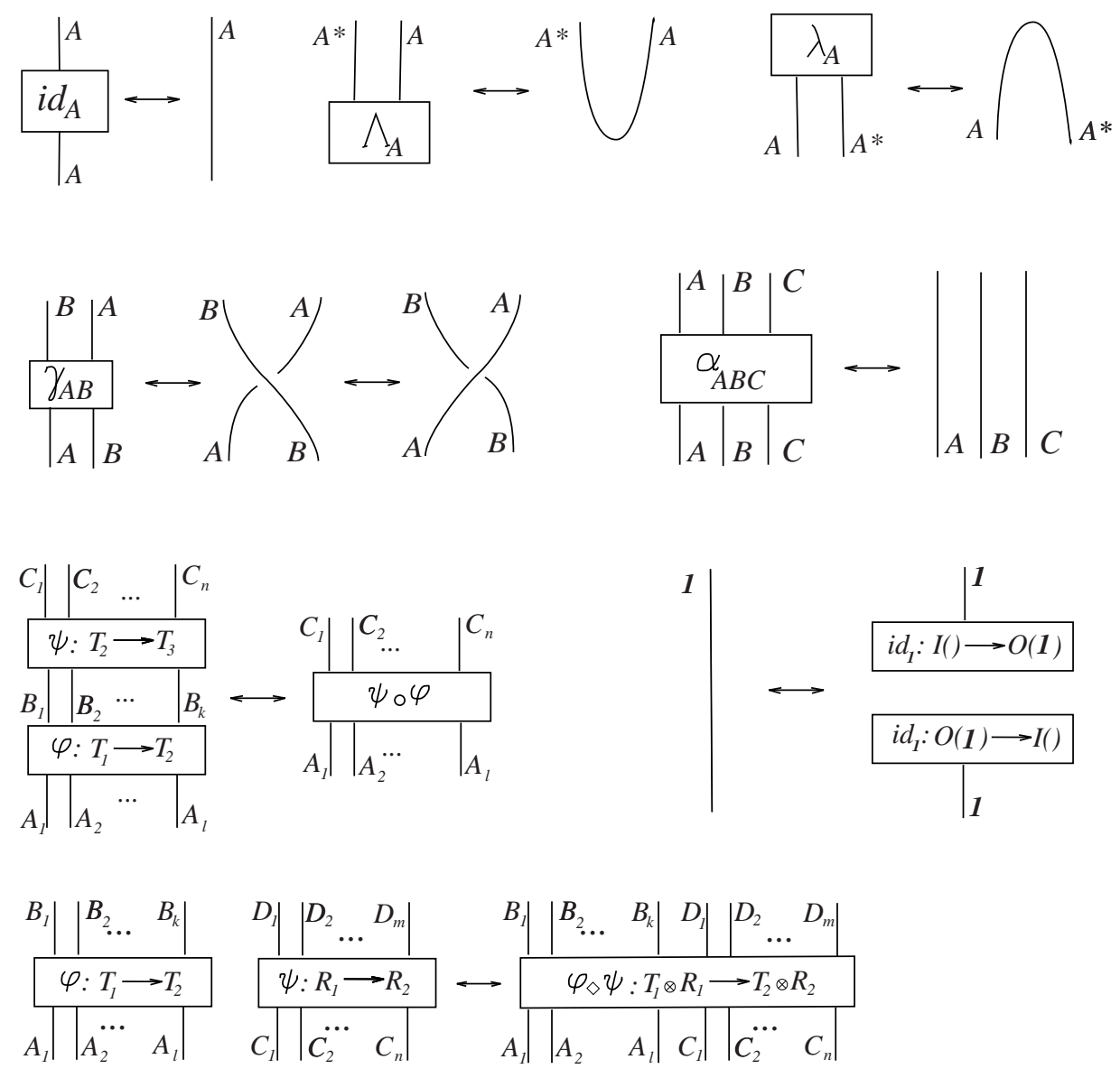

Figure 2. Diagram presentation of the relation 3.3

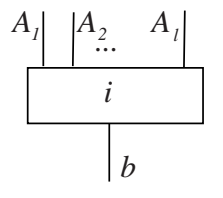

(a)

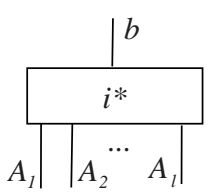

$A_{l}|| \begin{array}{ll}A_{2} & A_{l}\end{array} \mid$

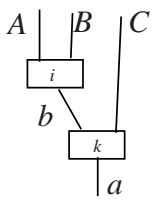

(c)

Figure 3. Diagram presentation of basic elements.

Proposition 3.9. $\left\{\zeta_{i}\left(c, b^{*} a\right)\right\}_{i}$ forms a basis for $F\left(c, b^{*} a\right)$ with a dual basis given by $\zeta_{i}\left(c, b^{*} a\right)^{*}=\frac{r(c)}{r(a)} \bar{\zeta}_{i}\left(c, b^{*} a\right)$. In particular, $\sum_{c, i} \frac{r(c)}{r(a)}\left(\zeta_{i}\left(c, b^{*} a\right) \circ \bar{\zeta}_{i}\left(c, b^{*} a\right)\right)=$ $i d_{b^{*} a}$.

The proof is presented in figure 7 .

3.10. To make it easier in the future to construct diagrams and to study parts of them, we introduce the notion of an open morphism, correspondingly open diagrams. An open morphism in $\mathbf{C} \mathcal{A}$ is a morphism $(F, \theta): \underline{A}=\left(A_{1}, A_{2}, \ldots, A_{l}\right) \rightarrow \underline{B}=$ 


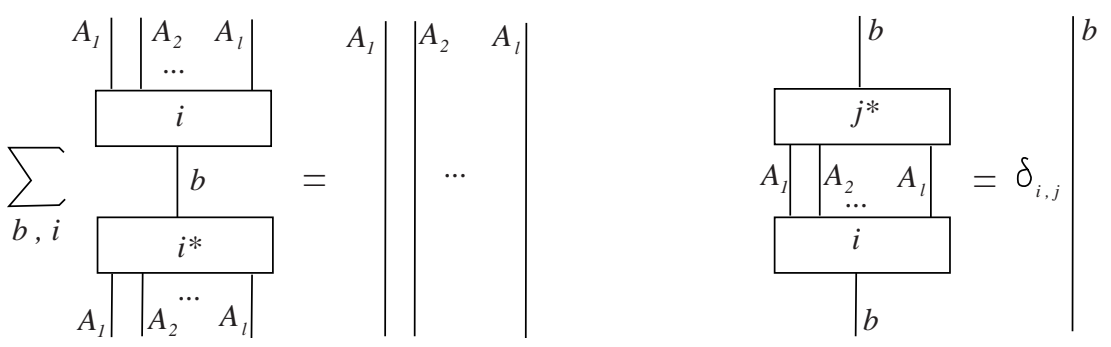

FiguRE 4. Diagram presentation of the defining identities for the dual basis.

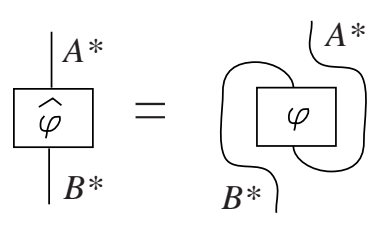

FiguRE 5. Diagram presentation of $\hat{\varphi}$.

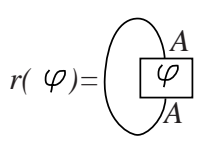

(a)

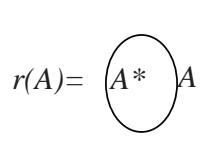

(b)



(c)

FiguRE 6. Rank of a morphism and rank of an object.

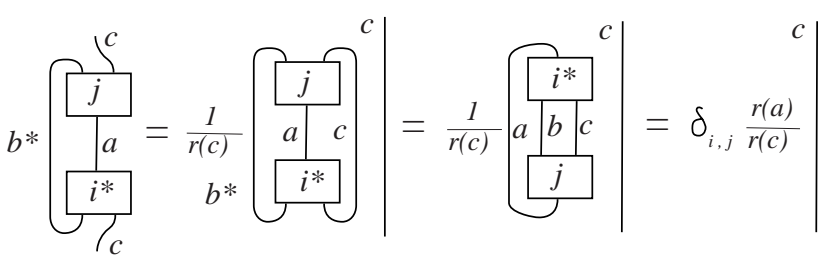

Figure 7. Proof of proposition 3.9.

$\left(B_{1}, B_{2}, \ldots, B_{k}\right)$ where some subset $\operatorname{In}(F)$ of the lower ends is labeled as $i n$-ends, and some subset $\operatorname{Out}(F)$ of the upper ends is labeled as out-ends. We refer to the set of $i n$ - and out- ends as free ends.

If $(F, \theta): \underline{A} \rightarrow \underline{B}$ and $(G, \xi): \underline{C} \rightarrow \underline{D}$ are two open morphisms, and $S \subset$ $\operatorname{Out}(F) \cap \operatorname{In}(G)$, we define the product of the two morphisms over $S$ to be the open morphism obtained by mapping the out-ends of $(F, \theta)$ labeled by $S$ into the in-ends of $(G, \xi)$ labeled by $S$ as shown on figure 8 (a). To be more precise, let $\underline{B}^{\prime}=\underline{B} \backslash S$ and $\underline{C}^{\prime}=\underline{C} \backslash S$. Then $(F, \theta) \circ_{S}(G, \xi): \underline{A} \otimes \underline{C}^{\prime} \rightarrow \underline{B}^{\prime} \otimes \underline{D}$ with $\operatorname{In}\left(G \circ_{S} F\right)=\operatorname{In}(F) \sqcup(\operatorname{In}(G) \backslash S)$ and $O u t\left(G \circ_{S} F\right)=(O u t(F) \backslash S) \sqcup O u t(G)$ is the following composition in $\mathbf{C} \mathcal{A}$ :

$$
(F, \theta) \circ_{S}(G, \xi)=\left(i d_{\underline{B}^{\prime}} \otimes(G, \xi)\right) \circ(\{\}, \epsilon) \circ\left((F, \theta) \otimes i d_{\underline{C}^{\prime}}\right),
$$

where $\epsilon: \underline{B} \sqcup \underline{C}^{\prime} \rightarrow\left(\underline{B}^{\prime} \sqcup \underline{C}\right)^{*}$ is the involution map sending $S \subset \underline{B} \rightarrow(S \subset \underline{C})^{*}$. 


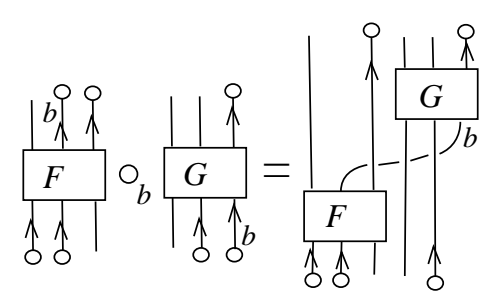

(a)

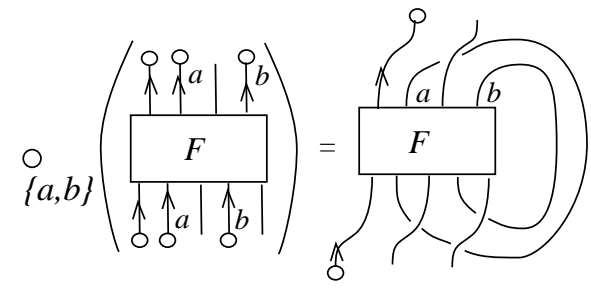

(b)

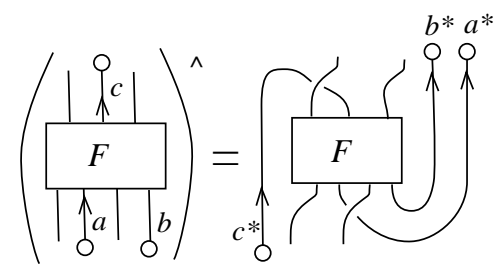

(c)

Figure 8. Product, closure and reverse of open morphisms.

In the diagrams we indicate the $i n$-ends with a circle and an arrow which comes out of it, and the out-ends with a circle and an arrow which goes into it. We will think of an open diagram as a sub-diagram of a bigger one, obtained by cutting out in the places of the free ends. For this reason, often the free ends will be left hanging without putting them in a specific place among the lower or the upper ends of the diagram, indicating in this way that the only important thing is how these will glue with the free ends of other open diagrams in the final "closed" one.

3.11. Given an open morphism $(F, \theta): \underline{A} \rightarrow \underline{B}$ we define the closure and the reverse of $(F, \theta)$. Given $S \subset \operatorname{In}(F) \cap O u t(F)$, the closure of $(F, \theta)$ over $S$ is defined to be the open morphisms $\circ_{S}(F, \theta)=\left(F, \theta^{\prime}\right)$, where $\theta^{\prime}$ is the extension of $\theta$ obtained by mapping into each other $S \subset \underline{A}$ and $S^{*} \subset \underline{B}^{*}$ as shown in figure $\mathbf{\theta}$ (b). By definition $\operatorname{In}\left(\circ_{S}(F)\right)=\operatorname{In}(F) \backslash S$ and $\operatorname{Out}\left(\circ_{S}(F)\right)=\operatorname{Out}(F) \backslash S$.

The reverse of $(F, \theta)$ is defined to be a morphism $(F, \theta)^{*}=(\hat{F}, \hat{\theta}):$ Out $(F)^{*} \otimes(\underline{A} \backslash$ $\operatorname{In}(F)) \rightarrow(\underline{B} \backslash \operatorname{Out}(F)) \otimes \operatorname{In}(F)^{*}$ with $\operatorname{In}(\hat{F})=\operatorname{Out}(F)^{*}$ and $\operatorname{Out}(\hat{F})=\operatorname{In}(F)^{*}$. $(F, \theta)$ is obtained from $(F, \theta)$ by exchanging the places of the in- and out- ends through composition with the corresponding form and coform in $\mathbf{C A}$ as it is shown in figure 8 (c).

\section{Partial traces}

4.1. Given a homomorphism $\phi: T\left(A_{1}, A_{2}, \ldots, A_{l}\right) \rightarrow T\left(A_{1}, A_{2}, \ldots, A_{l}\right), b \in \Sigma$ and a basis $\left\{\epsilon_{i}\right\}_{i}$ of $F\left(b, A_{k}\right)$, the partial trace of $\phi$ as $A_{k}$ goes to $b$ is defined to be the morphism

$$
\begin{aligned}
& \operatorname{Tr}_{A_{k} \rightarrow b}^{\epsilon_{i}}(\phi)=\sum_{i}\left(i d \diamond \epsilon_{i}^{*} \diamond i d\right) \circ \phi \circ\left(i d \diamond \epsilon_{i} \diamond i d\right): \\
& \quad T\left(A_{1}, \ldots, A_{k-1}, b, A_{k+1}, \ldots, A_{l}\right) \rightarrow T\left(A_{1}, \ldots, A_{k-1}, b, A_{k+1}, \ldots, A_{l}\right),
\end{aligned}
$$

where $\left\{\epsilon_{i}^{*}\right\}_{i}$ is the dual basis of $F\left(A_{k}, b\right)$, and $(i d \diamond \psi \diamond i d)$ stands for $T\left(i d_{A_{1}}, \ldots, i d_{A_{k-1}}, \psi, i d_{A_{k+1}}, \ldots, i d_{A_{l}}\right)$. The corresponding diagram is presented in figure 9 (a). 


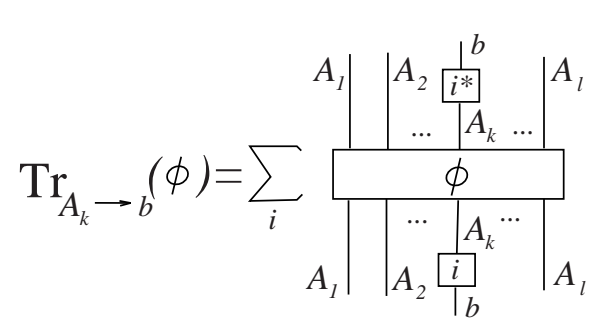

(a)

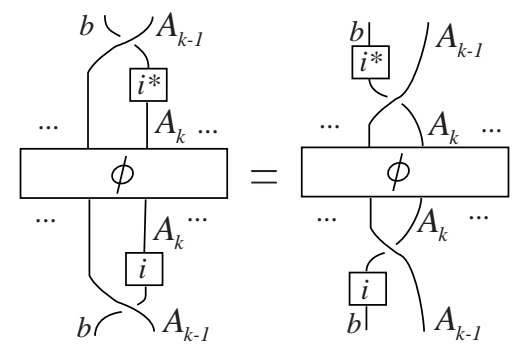

(b)

Figure 9. Partial trace.

4.2. Let $\phi$ be as above.

(a) If $\psi: A_{k} \rightarrow A_{k}^{\prime}$ is an isomorphism and $\epsilon_{i}, \epsilon_{i}^{\prime}$ are bases of $F\left(b, A_{k}\right)$ and $F\left(b, A_{k}^{\prime}\right)$ correspondingly, then

$$
\operatorname{Tr}_{A_{k} \rightarrow b}^{\epsilon_{i}}(\phi)=\operatorname{Tr}_{A_{k}^{\prime} \rightarrow b}^{\epsilon_{i}^{\prime}}\left((i d \diamond \psi \diamond i d) \circ \phi \circ\left(i d \diamond \psi^{-1} \diamond i d\right)\right) ;
$$

(b) If $k \neq m$, and $a, b \in \Sigma$, then

$$
\operatorname{Tr}_{A_{m} \rightarrow a}\left(\operatorname{Tr}_{A_{k} \rightarrow b}(\phi)\right)=\operatorname{Tr}_{A_{k} \rightarrow b}\left(\operatorname{Tr}_{A_{m} \rightarrow a}(\phi)\right) \equiv \operatorname{Tr}_{\left\{A_{k} \rightarrow b, A_{m} \rightarrow a\right\}}(\phi) ;
$$

(c) If $A_{k}=R\left(B_{1}, B_{2}, \ldots, B_{m}\right)$, i.e. for some $T^{\prime}$,

$T\left(A_{1}, A_{2}, \ldots, A_{l}\right)=T^{\prime}\left(A_{1}, A_{2}, \ldots, A_{k-1}, B_{1}, \ldots, B_{m}, A_{k+1}, \ldots, A_{l}\right)$,

then

$$
\operatorname{Tr}_{A_{k} \rightarrow b}(\phi)=\sum_{a \in \Sigma} \operatorname{Tr}_{R\left(B_{1}, \ldots, B_{s-1}, a, B_{s+1}, \ldots, B_{m}\right) \rightarrow b}\left(\operatorname{Tr}_{B_{s} \rightarrow a}(\phi)\right) ;
$$

(d) If $\pi$ is a permutation in $\mathbf{S}_{l}$, then $\pi \circ \operatorname{Tr}_{A_{k} \rightarrow b}(\phi) \circ \pi^{-1}=\operatorname{Tr}_{A_{\pi(k)} \rightarrow b}\left(\pi \circ \phi \circ \pi^{-1}\right)$.

(a) follows from the semisimplicity of the category in the following way:

$$
\begin{aligned}
& \sum_{i}\left(i d \diamond\left(\epsilon_{i}^{\prime *} \circ \psi\right) \diamond i d\right) \circ \phi \circ\left(i d \diamond\left(\psi^{-1} \circ \epsilon_{i}^{\prime}\right) \diamond i d\right)= \\
& =\sum_{i, j}\left(i d \diamond\left(\epsilon_{i}^{\prime *} \circ \psi \circ \epsilon_{j}\right) \diamond i d\right) \circ\left(i d \diamond \epsilon_{j}^{*} \diamond i d\right) \circ \phi \circ\left(i d \diamond\left(\psi^{-1} \circ \epsilon_{i}^{\prime}\right) \diamond i d\right)= \\
& =\sum_{i, j}\left(i d \diamond \epsilon_{j}^{*} \diamond i d\right) \circ \phi \circ\left(i d \diamond\left(\psi^{-1} \circ \epsilon_{i}^{\prime}\right) \diamond i d\right) \circ\left(i d \diamond\left(\epsilon_{i}^{\prime *} \circ \psi \circ \epsilon_{j}\right) \diamond i d\right)= \\
& =\sum_{j}\left(i d \diamond \epsilon_{j}^{*} \diamond i d\right) \circ \phi \circ\left(i d \diamond \epsilon_{j} \diamond i d\right) .
\end{aligned}
$$

(b) follows directly from the definition. To prove (c), we observe that from 2.6 follows that if $\left\{\tau_{i}\right\}_{i}$ is a basis for $F\left(b, R\left(B_{1}, \ldots, B_{s-1}, a, B_{s+1}, \ldots, B_{m}\right)\right)$, and $\left\{\epsilon_{j}\right\}_{j}$ is a basis for $F\left(a, B_{s}\right)$, then $\left\{R\left(i d_{B_{1}}, \ldots, i d_{B_{s-1}}, \epsilon_{j}, i d_{B_{s+1}} \ldots, i d_{B_{m}}\right) \circ \tau_{i}\right\}_{a, i, j}$ is a basis for $F\left(b, A_{k}\right)$. The use of this basis in the evaluation of the partial trace leads to the expression in (c). The statement in $(\mathrm{d})$ is straightforward: every permutation is a product of ones of the form $(k-1, k)$, and for those is shown in figure 9 (b).

As a consequence of (a) above, the indication of the basis in the notation of the partial trace will be omitted. 


\section{Definition of the invariant}

To simplify the notation, we make a standard choice of bracketing. When $T$ is of the form $(\ldots((O \otimes O) \otimes O) \cdots \otimes O) \otimes O$, we will write $T\left(B^{k}\right)$ as simply $B^{k}$, and $T\left(B_{1}, B_{2}, \ldots, B_{k}\right)$ as $B_{1} \diamond B_{2} \diamond \cdots \diamond B_{k}$. In particular, $A(B C) B^{3} C=$ $(((A,(B C))((B, B), B)) C$.

5.1. We start by introducing some definitions. Given any $b \in \Sigma$ define

$$
b^{l} \equiv\left\{\begin{array}{c}
b^{l} \text { if } l>0 \\
\left(b^{*}\right)^{|l|} \text { if } l<0
\end{array}\right.
$$

Then if $l>0$, let $f(b, l)=i d_{b^{l+1}}:(b, b, \ldots, b) \rightarrow(b, b, \ldots, b)$ be the open morphism in $\mathbf{C A}$, where $\operatorname{In}(f(b, l))$ is the first copy of $b$ in the lower ends and $\operatorname{Out}(f(b, l))$ is the last copy of $b$ among the upper ends. For $l<0$, we define $f(b, l)=f\left(b^{*},|l|\right)$ : $\left(b, b^{*}, \ldots, b^{*}\right) \rightarrow\left(b^{*}, \ldots, b^{*}, b\right)$. The diagrams corresponding to $f(b, l)$ in both cases are presented in figures 10 (a) and (b). Note that with this definition, for any integer $l, f(b, l)^{\wedge}=f\left(b^{*},-l\right)$.

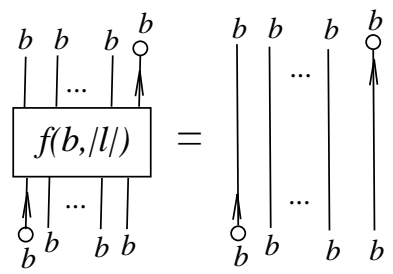

(a)

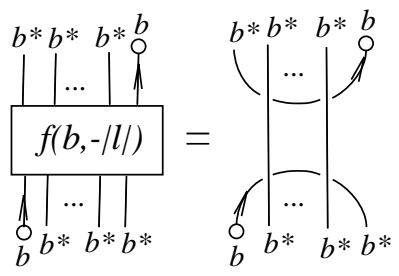

(b)

Figure 10. Diagram presentation of $f(b, l)$.

5.2. Suppose we are given a nonzero integer $l$ and $b_{1}, b_{2}, a_{i} \in \Sigma, 1 \leq i \leq|l|$. Let $\chi: b_{1} b_{1}^{l} b_{2} b_{2}^{l} \rightarrow\left(b_{1} b_{2}\right)\left(\diamond_{i}\left(b_{1} b_{2}\right)_{i}\right)$ and $\chi^{\prime}: b_{1}^{l} b_{1} b_{2}^{l} b_{2} \rightarrow\left(b_{1} b_{2}\right)\left(\diamond_{i}\left(b_{1} b_{2}\right)_{i}\right)$ be the corresponding permutations, where

$$
\left(b_{1} b_{2}\right)_{i}=\left\{\begin{array}{c}
b_{1} b_{2} \text { if } l>0 \\
b_{1}^{*} b_{2}^{*} \text { if } l<0
\end{array} .\right.
$$

Then $\operatorname{Tr}_{\left\{\left(b_{1} b_{2}\right)_{i} \rightarrow a_{i}\right\}_{i}}\left(\chi^{\prime} \circ\left(f\left(b_{1}, l\right) \otimes f\left(b_{2}, l\right)\right) \circ \chi^{-1}\right): b_{1} b_{2}\left(\diamond_{i} a_{i}\right) \rightarrow b_{1} b_{2}\left(\diamond_{i} a_{i}\right)$ is zero unless $a_{i}$ are not all the same, in which case is closely related to $f\left(a_{1}, l\right)$. To be able to give the precise statement, let $\left\{\epsilon_{k}\left(a_{i}, b_{1} b_{2}\right): a_{i} \rightarrow b_{1} b_{2}\right\}_{k}$ be a basis for $F\left(a_{i}, b_{1} b_{2}\right)$ and $\left.\left\{\epsilon_{k}\left(a_{i}, b_{1} b_{2}\right)\right)^{*}\right\}_{k}$ be its dual basis. We will denote with the same symbols the open morphism in $\mathbf{C} \mathcal{A}:\left(a_{i}\right) \rightarrow\left(b_{1}, b_{2}\right)\left(\left(b_{1}, b_{2}\right) \rightarrow\left(a_{i}\right)\right)$ which have a unique coupon labeled by $\epsilon_{k}\left(a_{i}, b_{1} b_{2}\right)$ (correspondingly $\left.\left(\epsilon_{k}\left(a_{i}, b_{1} b_{2}\right)\right)^{*}\right)$, and all lower ends are $i n$, and all upper ends are out. Let $f^{\epsilon}\left(a_{i}, l\right)=\sum_{k} \epsilon_{k}\left(a_{i}, b_{1} b_{2}\right)^{*} \circ_{a_{i}} f\left(a_{i}, l\right) \circ_{a_{i}} \epsilon_{k}\left(a_{i}, b_{1} b_{2}\right)$ : $b_{1} b_{2} a_{1}^{l} \rightarrow a_{1}^{l} b_{1} b_{2}$. Then if $l>0$ we have

$$
\operatorname{Tr}_{\left\{\left(b_{1} b_{2}\right)_{i} \rightarrow a_{i}\right\}_{i}}\left(\chi^{\prime} \circ\left(f\left(b_{1}, l\right) \otimes f\left(b_{2}, l\right)\right) \circ \chi^{-1}\right)=\sqcap_{i=1}^{l-1} \delta_{a_{i}, a_{i+1}} \sum_{k} \pi \circ f^{\epsilon}\left(a_{1}, l\right) .
$$

Here $\pi: a_{1}^{l} b_{1} b_{2} \rightarrow b_{1} b_{2} a_{1}^{l}$ is the corresponding permutation. The proof is shown in figure 11 . 


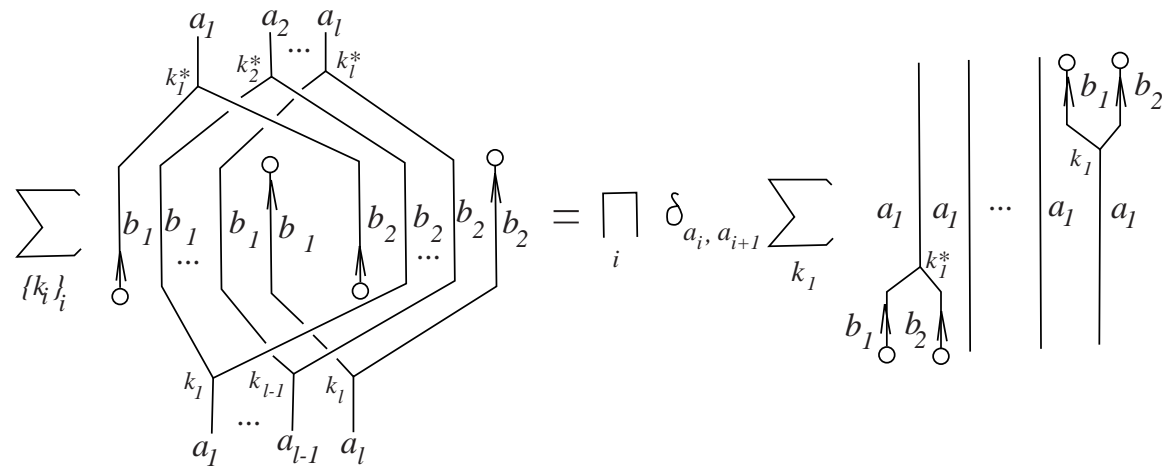

Figure 11. Proof of the property in 5.2 for positive $l$.

When $l<0$, the morphisms $\left\{\zeta_{k}\left(a_{i}^{*}, b_{1}^{*} b_{2}^{*}\right)=\gamma_{b_{2}^{*}, b_{1}^{*}} \circ \hat{\epsilon}_{k}\left(a_{i}, b_{1} b_{2}\right)\right\}_{k}$ form a basis for $F\left(a_{i}^{*}, b_{1}^{*} b_{2}^{*}\right)$. Then taking the partial trace with respect to this basis, also in this case, we obtain that

$$
\operatorname{Tr}_{\left\{\left(b_{1} b_{2}\right)_{i} \rightarrow a_{i}^{*}\right\}_{i}}\left(\chi^{\prime} \circ\left(f\left(b_{1}, l\right) \otimes f\left(b_{2}, l\right)\right) \circ \chi^{-1}\right)=\sqcap_{i=1}^{|l|-1} \delta_{a_{i}, a_{i+1}} \sum_{k} \pi \circ f^{\epsilon}\left(a_{1}, l\right) .
$$

The proof is shown in figure 12 .

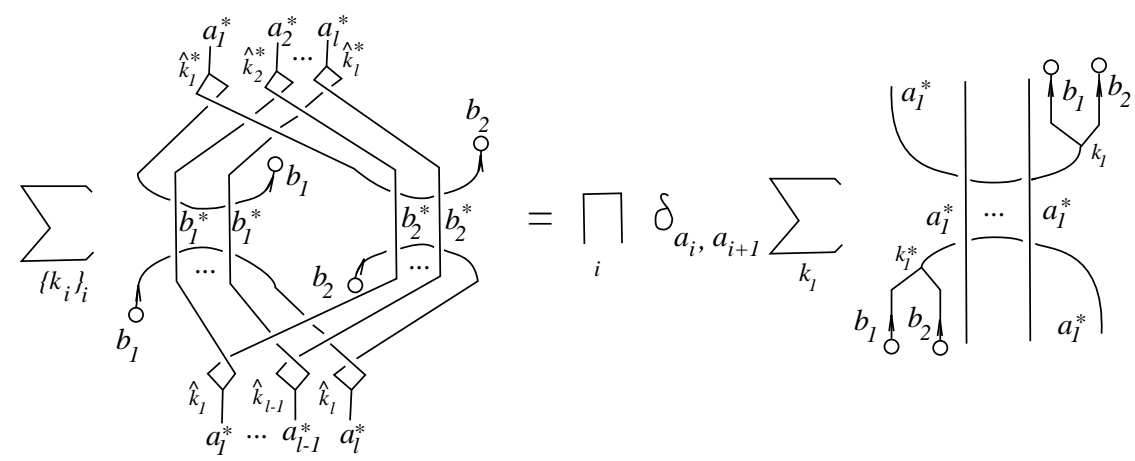

Figure 12. Proof of the property in 5.2 for negative $l$.

5.3. First we will define the invariant for the case of a presentation with one relation $P=<x_{1}, x_{2}, \ldots, x_{n} \mid R>$, where

$$
R=x_{i_{1}}^{l_{1}} x_{i_{2}}^{l_{2}} \ldots x_{i_{k}}^{l_{k}} .
$$

To the relation $R$ and to any $b \in \Sigma$ we associate an element $R(b)=b^{l_{1}} b^{l_{2}} \ldots b^{l_{k}} \in \mathcal{A}$ and a morphism $[R, b]: R(b) \rightarrow R(b)$ defined in the following way:

$$
[R, b]=\circ_{b}\left(r(b) f\left(b, l_{1}\right) \circ_{b} f\left(b, l_{2}\right) \circ_{b} \ldots \circ_{b} f\left(b, l_{k}\right)\right) .
$$

In other words, the out-end of $f\left(b, l_{i}\right)$ is connected with the $i n$-end of $f\left(b, l_{i+1}\right)$ and at the end the morphism is closed by connecting the out-end of $f\left(b, l_{k}\right)$ with the $i n$-end of $f\left(b, l_{1}\right)$. The result is multiplied by the rank of $b$. The open morphism without the final closure will be denoted with $[R, b]^{o}$. We will refer to the factors $b^{l}$ 
in $R(l)$ as $b$-factors. As examples, the diagrams corresponding to $\left[x^{2} y^{-2} x^{-1} y, b\right]^{o}$ and $\left[x^{2} y^{-2} x^{-1} y, b\right]$ are presented in figure 13 (a) and (b).



(a) $\left[x^{2} y^{-2} x^{-1} y, b\right]^{o}$
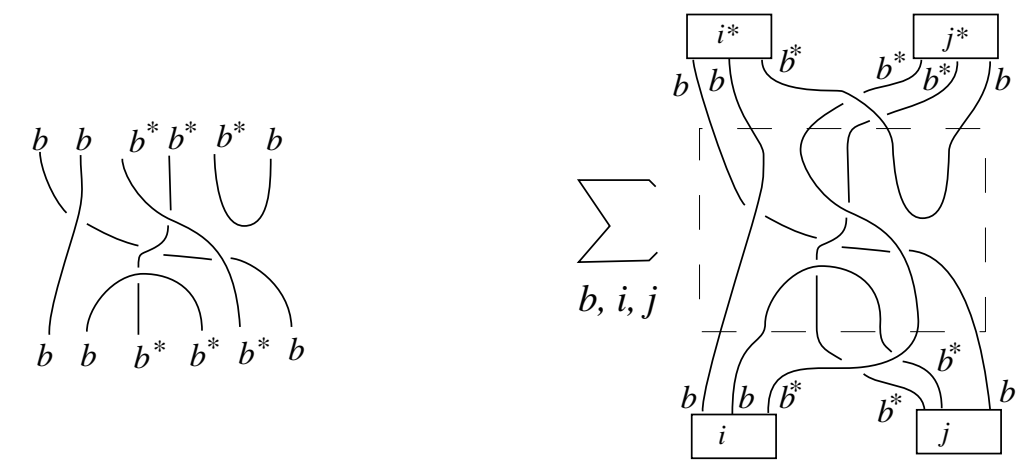

(b) $\left[x^{2} y^{-2} x^{-1} y, b\right]$

(c) $Q\left(<x, y \mid x^{2} y^{-2} x^{-1} y>\right)$

FiguRE 13. Diagram presentation of $Q\left(<x, y \mid x^{2} y^{-2} x^{-1} y x>\right)$.

5.4. Now to the relation $R$, to $b \in \Sigma$, and to any generator $x_{k}$, we associate an element $g_{k}(R, b) \in \mathcal{A}$, defined as the product of all b-factors in $R(b)$ corresponding to the generator $x_{k}: g_{k}(R, b)=\diamond_{r \mid x_{i_{r}}=x_{k}} b^{l_{r}}$. Let $\kappa(R): R(b) \rightarrow$ $g_{1}(R, b) g_{2}(R, b) \ldots g_{n}(R, b)$ be the permutation of factors. Then the invariant of the presentation $P$ is defined to be:

$$
Q(P)=\sum_{b \in \Sigma} \operatorname{Tr}_{\left\{g_{k}(R, b) \rightarrow \mathbf{1}\right\}_{k}}\left(\kappa(R) \circ[R, b] \circ \kappa(R)^{-1}\right) .
$$

The diagram corresponding to $Q\left(<x, y \mid x^{2} y^{-2} x^{-1} y>\right)$ is presented in figure 13 (c). The evaluation of the invariant for some simple examples is presented on figures 14 and the results are collected below.

(a) $Q\left(<x \mid x^{k}>\right)=\sum_{b \in \Sigma} r(b) \operatorname{Trace}\left[\rho_{\mathbf{1}}\left[b^{k}\right](1,2,3, \ldots, k)\right]$. In particular, $Q\left(<x \mid x^{2}>\right)=\sum_{b \in \Sigma \mid b=b^{*}} r(b) ;$

(b) The invariant for the standard presentation of the fundamental group of a surface of genus $n$ is $Q\left(<x_{1}, x_{2}, \ldots, x_{2 n} \mid \sqcap_{j=1}^{n-1} x_{2 j} x_{2 j+1} x_{2 j}^{-1} x_{2 j+1}^{-1}>\right)=$ $\sum_{b \in \Sigma} r(b)^{-2(n-1)}$

(c) $Q\left(<x, y \mid x y x y^{-1} x^{-1} y^{-1}>\right)=\sum_{b \in \Sigma \mid b=b^{*}} \operatorname{Trace}\left[\rho_{\mathbf{1}}\left[b^{3}\right](1,2,3)\right]$;

(d) $Q\left(<x, y \mid x y x^{-1} y>\right)=\left|\left\{b \in \Sigma \mid b=b^{*}\right\}\right|$.

Here Trace denotes the usual trace of the corresponding element in the representation of the symmetric group. 


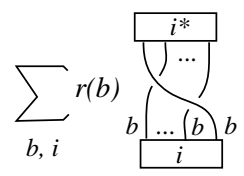

(a) $Q\left(<x \mid x^{k}>\right)$

$$
\begin{aligned}
& \sum_{b, i} r(b) \underbrace{i_{b}^{*}}_{\substack{b \prod_{b} \\
i}}=\sum_{b \mid b=b^{*}} r(b) \\
& Q\left(<x \mid x^{2}>\right)
\end{aligned}
$$

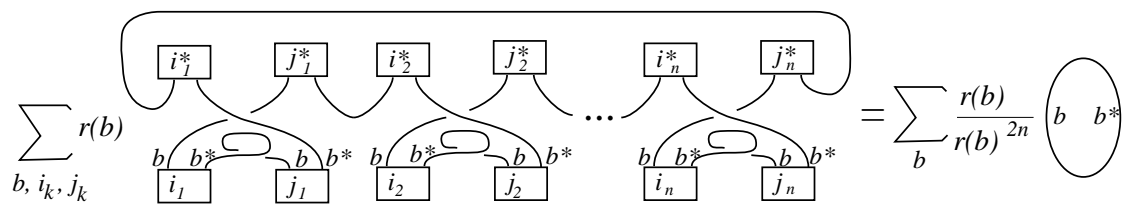

(b) $Q\left(<x_{1} x_{2}, \ldots, x_{2 n} \mid \prod_{i=1}^{n} x_{2 i-1} x_{2 i}^{-l} x_{2 i-1} x_{2 i}^{-1}>\right)$

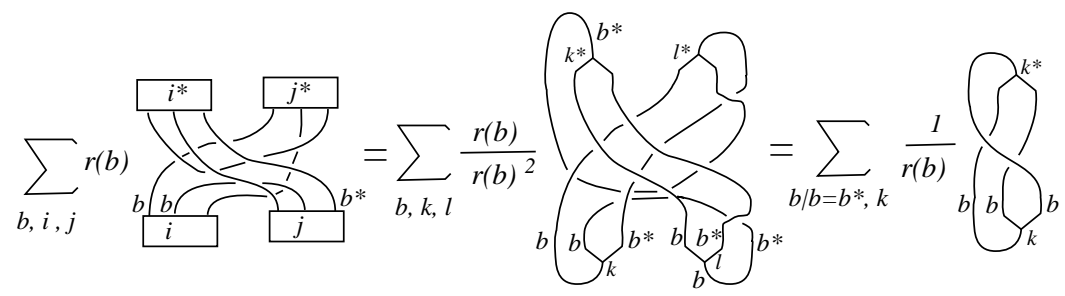

(c) $Q\left(<x\left|x y x y^{-1} x^{-1} y^{-1}\right\rangle\right)$

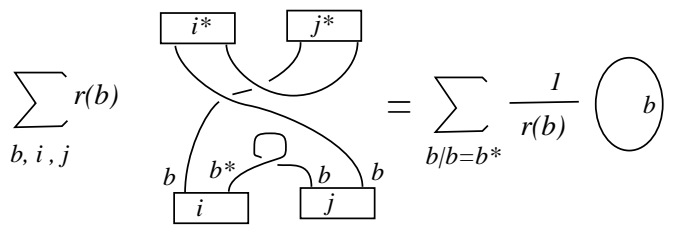

(d) $Q\left(\left\langle x \mid x y x^{-1} y\right\rangle\right)$

Figure 14. Some examples.

5.5. Now we extend the definition to the case of presentations with more then one relation. Let $P=<x_{1}, x_{2}, \ldots, x_{n} \mid R_{1}, R_{2}, \ldots, R_{m}>$ be such a presentation, and let $\underline{b}=\left(b_{1}, b_{2} \ldots b_{m}\right) \in \Sigma^{\times m}$. Then define

$$
\begin{aligned}
& \operatorname{Rel}(\underline{b})=\diamond_{j=1}^{m} R\left(b_{j}\right), \\
& {[P, \underline{b}]=\left[R_{1}, b_{1}\right] \diamond\left[R_{2}, b_{2}\right] \diamond \ldots \diamond\left[R_{m}, b_{m}\right]: \operatorname{Rel}(\underline{b}) \rightarrow \operatorname{Rel}(\underline{b}),} \\
& G_{k}(\underline{b})=\diamond_{j=1}^{m} g_{k}\left(R_{1}, b_{1}\right) g_{k}\left(R_{2}, b_{2}\right) \ldots g_{k}\left(R_{m}, b_{m}\right), \\
& \xi(P): \operatorname{Rel}(\underline{b}) \rightarrow \diamond_{k=1}^{n} G_{k}(\underline{b}) \text { to be the corresponding permutation of } b \text {-factors. }
\end{aligned}
$$

Note that the permutation $\xi(P)$ groups the $b$-factors in $\operatorname{Rel}(\underline{b})$ according to the generator to which they correspond. Then the invariant of the presentation $P$ is defined to be

$$
Q(P)=\sum_{\underline{b} \in \Sigma^{\times m}} \operatorname{Tr}_{\left\{G_{k}(\underline{b}) \rightarrow \mathbf{1}\right\}_{k}}\left(\xi(P) \circ[P, \underline{b}] \circ \xi(P)^{-1}\right) .
$$


As example the diagram corresponding to $Q\left(<x, y \mid x^{2} y^{2}, x y x^{-1} y^{-1}>\right)$ is shown in figure 15.

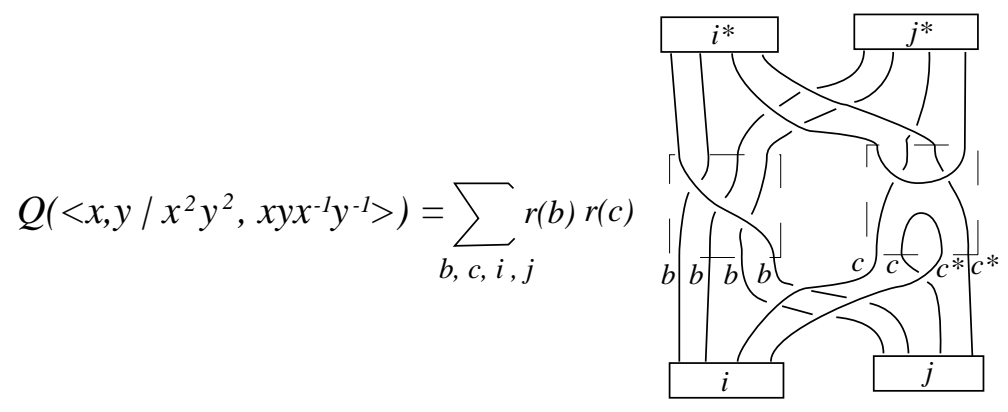

FiguRE 15. Example with two relations.

Theorem 5.6. $Q(P)$ is invariant under the AC-moves (i)-(vi).

The proof is contained in the next section.

\section{Invariance under the AC - moves}

We consider each move separately. The presentation obtained after performing the move will be denoted with $P^{\prime}$ and the corresponding products of $b$-factors will be denoted with $\operatorname{Rel}^{\prime}(\underline{b})$ and $G_{k}^{\prime}(\underline{b})$.

6.1. The first move consists in exchanging the places of $R_{1}$ and $R_{s}$. Let

$$
\underline{b}^{\prime}=\left(b_{s}, b_{2}, b_{3}, \ldots, b_{s-1}, b_{1}, b_{s+1}, \ldots, b_{m}\right)
$$

and let $\pi_{k}: G_{k}(\underline{b}) \rightarrow G_{k}^{\prime}\left(\underline{b}^{\prime}\right)$ be the permutation which exchanges the places of $g_{k}\left(R_{1}, b_{1}\right)$ and $g_{k}\left(R_{s}, b_{s}\right)$. Then $\pi=\pi_{1} \diamond \pi_{2} \diamond \ldots \diamond \pi_{n}: \diamond_{k=1}^{n} G_{k}(\underline{b}) \rightarrow \diamond_{k=1}^{n} G_{k}^{\prime}\left(\underline{b}^{\prime}\right)$. As morphisms in $\mathbf{C} \mathcal{A}$ we have that

$$
\pi \circ \xi(P) \circ[P, \underline{b}] \circ \xi(P)^{-1} \circ \pi^{-1}=\xi\left(P^{\prime}\right) \circ\left[P^{\prime}, \underline{b}^{\prime}\right] \circ \xi\left(P^{\prime}\right)^{-1} .
$$

This fact is illustrated in figure 16. Then according to 4.2 (a) we have

$$
\begin{aligned}
Q\left(P^{\prime}\right) & =\sum_{\underline{b}^{\prime} \in \Sigma^{\times m}} \operatorname{Tr}_{\left\{G_{k}^{\prime}\left(\underline{b}^{\prime}\right) \rightarrow \mathbf{1}\right\}_{k}}\left(\pi \circ \xi(P) \circ[P, \underline{b}] \circ \xi(P)^{-1} \circ \pi^{-1}\right)= \\
& =\sum_{\underline{b}^{\prime} \in \Sigma^{\times m}} \operatorname{Tr}_{\left\{G_{k}(\underline{b}) \rightarrow \mathbf{1}\right\}_{k}}\left(\xi(P) \circ[P, \underline{b}] \circ \xi(P)^{-1}\right)=Q(P) .
\end{aligned}
$$

This shows the invariance under the first move.

6.2. The second move conjugates $R_{1}$ with an arbitrary element in the group. Obviously, it is enough to show the invariance under conjugation with one of the generators $R_{1} \rightarrow R_{1}^{\prime}=x_{l} R_{1} x_{l}^{-1}$. Then $G_{k}$ changes only when $k=l$, and $G_{l}^{\prime}(\underline{b})=$ $b_{1} g_{l}\left(R_{1}, b_{1}\right) b_{1}^{*} g_{l}\left(R_{2}, b_{2}\right) \ldots g_{l}\left(R_{m}, b_{m}\right)$. Let

$$
\pi:\left(\diamond_{k<l} G_{k}(\underline{b})\right) G_{l}^{\prime}(\underline{b})\left(\diamond_{k>l} G_{k}(\underline{b})\right) \rightarrow\left(\diamond_{k<l} G_{k}(\underline{b})\right) b_{1} b_{1}^{*} G_{l}(\underline{b})\left(\diamond_{k>l} G_{k}(\underline{b})\right)
$$

be the corresponding permutation of factors. The goal now is to show that

$$
E=\operatorname{Tr}_{G_{l}^{\prime}(\underline{b}) \rightarrow \mathbf{1}}\left(\xi\left(P^{\prime}\right) \circ\left[P^{\prime}, \underline{b}\right] \circ \xi\left(P^{\prime}\right)^{-1}\right)=\operatorname{Tr}_{G_{l}(\underline{b}) \rightarrow \mathbf{1}}\left(\xi(P) \circ[P, \underline{b}] \circ \xi(P)^{-1}\right),
$$




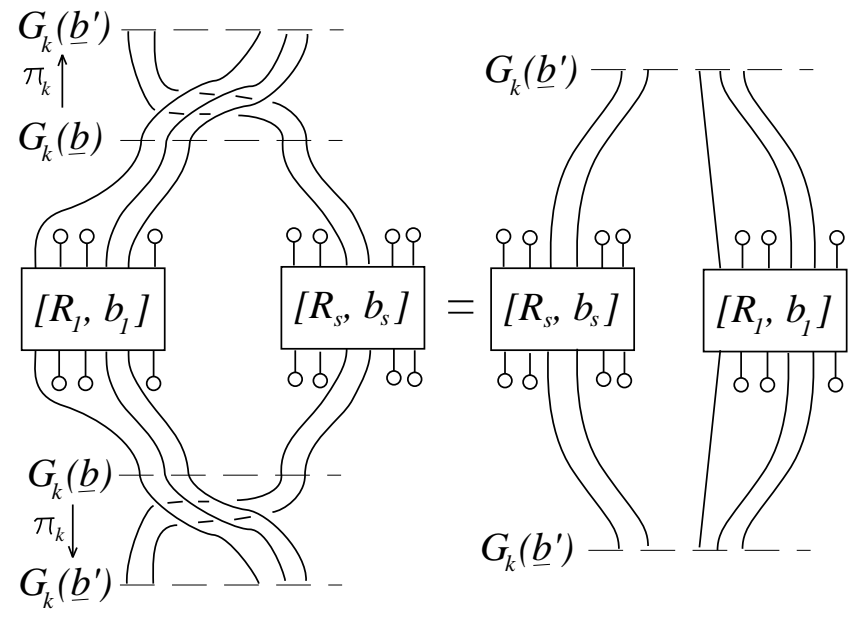

FiguRE 16. Invariance under move (i).

which according to 4.2 (b) would imply the statement. By using properties 4.2 (a) and (c) of the partial trace we obtain

$$
\begin{aligned}
E & =\operatorname{Tr}_{b_{1} b_{1}^{*} G_{l}(\underline{b}) \rightarrow \mathbf{1}}\left(\pi \circ \xi\left(P^{\prime}\right) \circ\left[P^{\prime}, \underline{b}\right] \circ \xi\left(P^{\prime}\right)^{-1} \circ \pi^{-1}\right)= \\
& =\sum_{a \in \Sigma} \operatorname{Tr}_{a G_{l}(\underline{b}) \rightarrow \mathbf{1}}\left[\operatorname{Tr}_{b_{1} b_{1}^{*} \rightarrow a}\left(\pi \circ \xi\left(P^{\prime}\right) \circ\left[P^{\prime}, \underline{b}\right] \circ \xi\left(P^{\prime}\right)^{-1} \circ \pi^{-1}\right)\right] .
\end{aligned}
$$

We remind that $\left[P^{\prime}, \underline{b}\right]=\diamond_{i=1}^{m}\left[R_{i}^{\prime}, b_{i}\right]$, but the partial trace $\operatorname{Tr}_{b_{1} b_{1}^{*} \rightarrow a}$ actually involves only the morphism $\left[R_{1}^{\prime}, b_{1}\right]$, in sense that $\left[R_{1}^{\prime}, b_{1}\right]$ maps $b_{1} R_{1}\left(b_{1}\right) b_{1}^{*}$ into itself, as shown on figure 17 (a), and the effect of the permutation $\pi$ is to move the $b$ factors $b_{1}$ and $b_{1}^{*}$ together, so that the partial trace can be taken. Then figure 17 (b) proves that

$\operatorname{Tr}_{b_{1} b_{1}^{*} \rightarrow a}\left(\pi \circ \xi\left(P^{\prime}\right) \circ\left[P^{\prime}, \underline{b}\right] \circ \xi\left(P^{\prime}\right)^{-1} \circ \pi^{-1}\right)=\delta_{a, \mathbf{1}} \nu \circ\left(i d_{\mathbf{1}} \diamond\left(\xi(P) \circ[P, \underline{b}] \circ \xi(P)^{-1}\right)\right) \circ \nu^{-1}$.

Here $\nu: 1 G_{1}(\underline{b}) G_{2}(\underline{b}) \ldots G_{2}(\underline{b}) \rightarrow G_{1}(\underline{b}) G_{2}(\underline{b}) \ldots G_{l-1}(\underline{b}) \mathbf{1} G_{l}(\underline{b}) \ldots G_{n}(\underline{b})$ is the corresponding permutation. The factor $\mathbf{1}$ can be removed from $\operatorname{Tr}_{G_{l}(\underline{b}) \rightarrow \mathbf{1}}$ by conjugating the argument with $\eta_{G_{l}(\underline{b})}$ and using 4.2 (a). This completes the proof of the invariance under (ii).

6.3. The third move consists of replacing $R_{1}$ with $R_{1}^{-1}$. First we observe that if $R=x_{i_{1}}^{l_{1}} x_{i_{2}}^{l_{2}} \ldots x_{i_{s}}^{l_{s}}$ is a relation, then

$$
\begin{aligned}
{\left[R^{-1}, b\right] } & =r(b) \circ_{b}\left(f\left(b,-l_{s}\right) \circ_{b} f\left(b,-l_{s-1}\right) \circ_{b} \ldots \circ_{b} f\left(b,-l_{1}\right)\right)= \\
& =r\left(b^{*}\right) \circ_{b}\left(f\left(b^{*}, l_{s}\right) \circ_{b} f\left(b^{*}, l_{s-1}\right) \circ_{b} \ldots \circ_{b} f\left(b^{*}, l_{1}\right)\right)= \\
& =\pi(R) \circ\left[R, b^{*}\right] \circ \pi(R)^{-1},
\end{aligned}
$$

where $\pi(R): b^{-l_{1}} b^{-l_{2}} \ldots b^{-l_{s}} \rightarrow b^{-l_{s}} b^{-l_{s-1}} \ldots b^{-l_{1}}$ is the permutation of $b$-factors. The last equality is illustrated in figure 18. In the case we want to study, $\pi\left(R_{1}\right)$ induces a permutation $\pi=\pi\left(R_{1}\right) \diamond i d: R_{1}\left(b_{1}^{*}\right) \diamond\left(\diamond_{i=2}^{m} R_{i}\left(b_{i}\right)\right) \rightarrow R_{1}^{-1}\left(b_{1}\right) \diamond\left(\diamond_{i=2}^{m} R_{i}\left(b_{i}\right)\right)$. Then from the discussion above it follows that

$$
\left[P^{\prime}, \underline{b}\right]=\pi \circ[P, \underline{\hat{b}}] \circ \pi^{-1},
$$


(a)

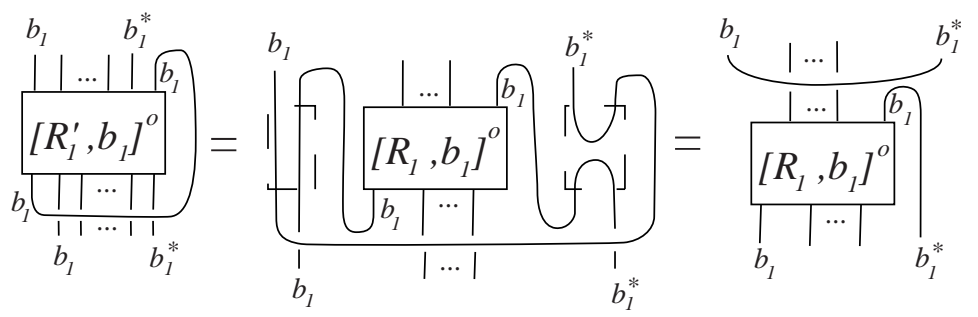

(b)



Figure 17. Invariance under move (ii).



FIgURE 18. Invariance under move (iii).

where $\underline{\hat{b}}=\left(b_{1}^{*}, b_{2}, \ldots, b_{m}\right)$. Moreover, $G_{k}^{\prime}(\underline{b})$ can be obtained from $G_{k}(\underline{\hat{b}})$ by reversing the order of the $b$-factors of the form $b_{1}^{l_{j}}$. Let the corresponding permutation be $\chi_{k}: G_{k}^{\prime}(\underline{b}) \rightarrow G_{k}(\underline{\hat{b}})$, and let $\chi=\chi_{1} \diamond \chi_{2} \diamond \ldots \diamond \chi_{m}: \diamond_{k=1}^{n} G_{k}^{\prime}(\underline{b}) \rightarrow \diamond_{k=1}^{n} G_{k}(\underline{\hat{b}})$. Then from property 4.2 we obtain

$$
\begin{aligned}
Q\left(P^{\prime}\right) & =\sum_{\underline{b} \in \Sigma^{\times m}} \operatorname{Tr}_{\left\{G_{k}^{\prime}(\underline{b}) \rightarrow \mathbf{1}\right\}_{k}}\left(\xi\left(P^{\prime}\right) \circ\left[P^{\prime}, \underline{b}\right] \circ \xi\left(P^{\prime}\right)^{-1}\right)= \\
& =\sum_{\underline{b} \in \Sigma^{\times m}} \operatorname{Tr}_{\left\{G_{k}(\underline{\hat{b}}) \rightarrow \mathbf{1}\right\}_{k}}\left(\chi \circ \xi\left(P^{\prime}\right) \circ \pi \circ[P, \underline{\hat{b}}] \circ \pi^{-1} \circ \xi\left(P^{\prime}\right)^{-1} \circ \chi^{-1}\right)= \\
& =\sum_{\underline{b} \in \Sigma^{\times m}} \operatorname{Tr}_{\left\{G_{k}(\underline{\hat{b}}) \rightarrow \mathbf{1}\right\}_{k}}\left(\xi(P) \circ[P, \underline{\hat{b}}] \circ \xi(P)^{-1}\right)=Q(P) .
\end{aligned}
$$

This completes the proof of the invariance under move (iii). 
6.4. The forth move consists of replacing $R_{1}$ with $R_{1} R_{2}$. Figure 19 presents the corresponding change in $[P, \underline{b}]$. Let $R_{2}=x_{i_{1}}^{l_{1}} l_{i_{2}}^{l_{2}} \ldots x_{i_{s}}^{l_{s}}$, and

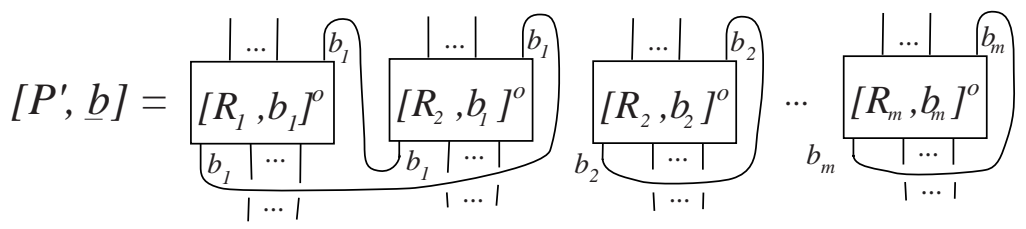

Figure 19. Change in $[P, \underline{b}]$ under move (iv).

$\chi: R_{1}\left(b_{1}\right) R_{2}\left(b_{1}\right) R_{2}\left(b_{2}\right) \rightarrow R_{1}\left(b_{1}\right) \diamond_{i=1}^{s} \diamond_{j=1}^{\left|l_{i}\right|}\left(b_{1} b_{2}\right)_{i, j}$ be the corresponding permutation, where

$$
\left(b_{1} b_{2}\right)_{i, j}=\left\{\begin{array}{l}
b_{1} b_{2} \text { if } l_{i}>0 \\
b_{1}^{*} b_{2}^{*} \text { if } l_{i}<0
\end{array} .\right.
$$

Then $\chi \circ\left(\left[R_{1} R_{2}, b_{1}\right] \diamond\left[R_{2}, b_{2}\right]\right) \circ \chi^{-1}$ maps $R_{1}\left(b_{1}\right) \diamond_{i=1}^{s} \diamond_{j=1}^{\left|l_{i}\right|}\left(b_{1} b_{2}\right)_{i, j}$ into itself. As a first step we prove that

$$
\begin{array}{r}
W=\sum_{b_{2} \in \Sigma} \operatorname{Tr}_{\left\{\left(b_{1} b_{2}\right)_{i, j} \rightarrow a_{i, j}\right\}_{i, j}}\left(\chi \circ\left(\left[R_{1} R_{2}, b_{1}\right] \diamond\left[R_{2}, b_{2}\right]\right) \circ \chi^{-1}\right)= \\
\Pi_{i=1}^{s-1}\left(\delta_{a_{i, 1}, a_{i+1,1}} \sqcap_{j=1}^{\left|l_{i}\right|-1} \delta_{a_{i, j}, a_{i, j+1}}\right)\left(\left[R_{1}, b_{1}\right] \diamond\left[R_{2}, a_{1,1}\right]\right)
\end{array}
$$

Let $a_{i} \equiv a_{i, 1}, 1 \leq i \leq s$. Recalling 5.1, where the partial trace involving $f\left(b_{1}, l\right) \otimes$ $f\left(b_{2}, l\right)$ was evaluated, and we have that $W=\circ_{b_{1}}\left(\left[R_{1}, b_{1}\right]^{o} \circ_{b_{1}} G\right)$, where

$G=\left(\sqcap_{i=1}^{s-1} \sqcap_{j=1}^{\left|l_{i}\right|-1} \delta_{a_{i, j}, a_{i, j+1}}\right) \sum_{b_{2} \in \Sigma} \circ_{b_{2}}\left(f^{\epsilon}\left(a_{1}, l_{1}\right) \circ_{\left\{b_{1}, b_{2}\right\}} f^{\epsilon}\left(a_{2}, l_{2}\right) \circ_{\left\{b_{1}, b_{2}\right\}} \cdots \circ_{\left\{b_{1}, b_{2}\right\}} f^{\epsilon}\left(a_{s}, l_{s}\right)\right)$.

Then the statement for $W$ follows from the fact that $\epsilon_{k}\left(a_{i}, b_{1} b_{2}\right)^{*} \circ \epsilon_{l}\left(a_{i+1}, b_{1} b_{2}\right)=$ $\delta_{a_{i}, a_{i+1}} \delta_{k, l} i d_{a_{i}}$ and from proposition 3.9 as it is shown in figure 20.

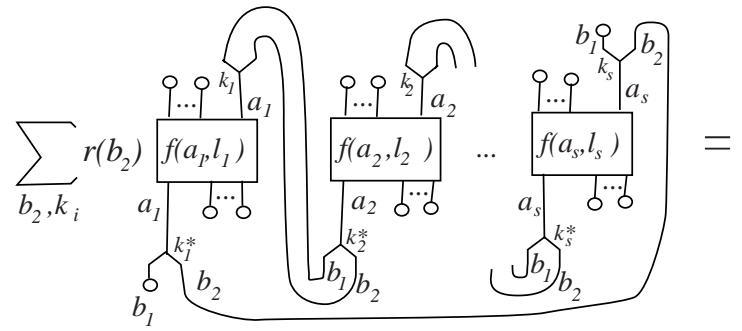

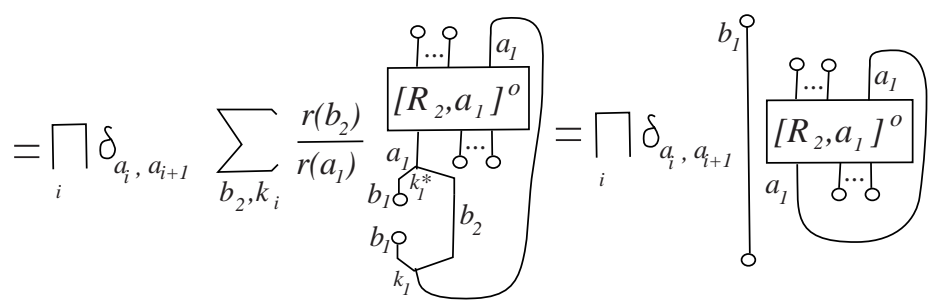

Figure 20. Invariance under move (iv).

Now the invariance under move (iv) is easy to show. Fix $k$ between 1 and $n$. Then after the move, $G_{k}^{\prime}(\underline{b})=g_{k}\left(R_{1}, b_{1}\right) g_{k}\left(R_{2}, b_{1}\right) g_{k}\left(R_{2}, b_{2}\right) \ldots g_{k}\left(R_{m}, b_{m}\right)$. Let 
$G_{k}^{>2}(\underline{b})=\diamond_{i=3}^{s} g_{k}\left(R_{i}, b_{i}\right)$ and $g_{k}\left(R_{2}, b\right)=\diamond_{r=1}^{s_{k}} b^{l_{i_{r}}}$. Then define $\tau_{k}: G_{k}^{\prime}(\underline{b}) \rightarrow$ $g_{k}\left(R_{1}, b_{1}\right) \diamond_{r=1}^{s_{k}} \diamond_{j=1}^{\left|l_{i_{r}}\right|}\left(b_{1} b_{2}\right)_{i_{r}, j} G_{k}^{>2}(\underline{b})$ to be the corresponding permutation, and let $\tau=\tau_{1} \diamond \tau_{2} \diamond \ldots \diamond \tau_{m}: \diamond_{k=1}^{n} G_{k}^{\prime}(\underline{b}) \rightarrow \diamond_{k=1}^{n} g_{k}\left(R_{1}, b_{1}\right) \diamond_{r=1}^{s_{k}} \diamond_{j=1}^{\left|l_{i_{r}}\right|}\left(b_{1} b_{2}\right)_{i_{r}, j} G_{k}^{>2}(\underline{b})$. Then from 4.2 (a) and (c) we obtain,

$$
\begin{aligned}
& \sum_{\underline{b} \in \Sigma^{\times m}} \operatorname{Tr}_{G_{k}^{\prime}(\underline{b}) \rightarrow \mathbf{1}}\left(\xi\left(P^{\prime}\right) \circ\left[P^{\prime}, \underline{b}\right] \circ \xi\left(P^{\prime}\right)^{-1}\right)= \\
& =\sum_{\underline{b} \in \Sigma^{\times m}} \operatorname{Tr} \underbrace{}_{g_{k}\left(R_{1}, b_{1}\right) \diamond_{r=1}^{s_{k}} \diamond_{\diamond_{j=1}}^{\left|l_{i_{r}}\right|}\left(b_{1} b_{2}\right)_{i_{r}, j} G_{k}^{>2}(\underline{b}) \rightarrow \mathbf{1}}\left(\tau \circ \xi\left(P^{\prime}\right) \circ\left[P^{\prime}, \underline{b}\right] \circ \xi\left(P^{\prime}\right)^{-1} \circ \tau^{-1}\right)= \\
& =\sum_{\underline{b} \in \Sigma^{\times m}} \sum_{\underline{a}_{r} \in \Sigma^{\times s_{k}}} \operatorname{Tr}_{g_{k}\left(R_{1}, b_{1}\right) \diamond_{r=1}^{s_{k}} \diamond_{j=1}^{\left|l_{i_{r} \mid}\right|} a_{r, j} G_{k}^{>2}(\underline{b}) \rightarrow \mathbf{1}} \operatorname{Tr}_{\left\{\left(b_{1} b_{2}\right)_{i_{r}, j} \rightarrow a_{r, j}\right\}_{r, j}} \\
& \left(\tau \circ \xi\left(P^{\prime}\right) \circ\left[P^{\prime}, \underline{b}\right] \circ \xi\left(P^{\prime}\right)^{-1} \circ \tau^{-1}\right) .
\end{aligned}
$$

Now we observe that

$\tau \circ \xi\left(P^{\prime}\right) \circ\left[P^{\prime}, \underline{b}\right] \circ \xi\left(P^{\prime}\right)^{-1} \circ \tau^{-1}=\xi(P) \circ\left(\left(\chi \circ\left(\left[R_{1} R_{2}, b_{1}\right] \diamond\left[R_{2}, b_{2}\right]\right) \circ \chi^{-1}\right) \diamond_{i=3}^{m}\left[R_{i}, b_{i}\right]\right) \circ \xi(P)^{-1}$.

Here the use of $\xi(P)$ is somewhat abusive. By definition $\xi(P)$ is the permutation of $b$-factors $\diamond_{j=1}^{m} R\left(b_{j}\right) \rightarrow \diamond_{k=1}^{n} G_{k}(\underline{b})$, while on the r.h.s. above the same notation is used to indicate the same permutation between expressions where each factors $b_{2}^{l_{i}}$ has been replaced with $\diamond_{j=1}^{l_{i}}\left(b_{1} b_{2}\right)_{i, j}$. Then the statement follows from $4.2(\mathrm{~d})$.

6.5. The invariance under the moves (v) and (vi) is straightforward: according to the definition, these moves change the value of the invariant by multiplication or division by

$$
\sum_{b \in \Sigma} r(b) \operatorname{Tr}_{b \rightarrow \mathbf{1}} i d_{b}=1
$$

\section{The definition of Quinn's invariant}

7.1. In this section we show that the invariant defined above is actually the one produced by the algorithm described in [8]. First we introduce some morphisms which are being used in the algorithm.

(i) Given any $a, b, c \in \Sigma$ define $\operatorname{cycl}(a, b, c): F(a, b c) \rightarrow F\left(b^{*}, c a^{*}\right)$ as

$$
\begin{aligned}
\operatorname{cycl}(a, b, c)(\phi): \quad & b^{*} \rightarrow b^{*} \mathbf{1} \stackrel{\Lambda_{a}^{*}}{\longrightarrow} b^{*}\left(a a^{*}\right) \stackrel{i d_{b^{*}} \diamond\left(\phi \diamond i d_{a^{*}}\right)}{\longrightarrow} \\
& b^{*}\left((b c) a^{*}\right) \rightarrow b^{*} b\left(c a^{*}\right) \stackrel{\lambda_{b^{*}}}{\longrightarrow} \mathbf{1}\left(c a^{*}\right) \rightarrow c a^{*} .
\end{aligned}
$$

$\operatorname{cycl}(a, b, c)$ is actually an isomorphisms with inverse $\operatorname{cycl}\left(c^{*}, a^{*}, b\right) \circ \operatorname{cycl}\left(b^{*}, c, a^{*}\right)$.

(ii) Given any $a, b \in \Sigma$ and $A \in \Sigma$ define

$$
\begin{aligned}
m_{b}: F(a, A) \longrightarrow F\left(\mathbf{1},\left(b b^{*}\right) A\right), & \psi \rightarrow\left(\Lambda_{b^{*}} \diamond \psi\right) \circ \eta_{a}{ }^{-1} \\
d_{b}: F\left(\mathbf{1},\left(b b^{*}\right) A\right) \longrightarrow F(a, A), & \phi \rightarrow \eta_{A} \circ\left(\lambda_{b} \diamond i d_{A}\right) \circ \phi .
\end{aligned}
$$

The corresponding diagrams are presented in figures 21.

7.2. The invariant of a presentation $P=\left(x_{1}, x_{2}, \ldots, x_{n} \mid R_{1}, R_{2}, \ldots, R_{m}\right)$ as defined in 8 is a map $Q(P): F(\mathbf{1}, \mathbf{1}) \rightarrow F(\mathbf{1}, \mathbf{1})$, i.e. an element in $F(\mathbf{1}, \mathbf{1}) \simeq K$. This map is obtained as a composition of the morphisms which are listed below. Here we will refer to the space $V(k)=\oplus_{y_{j} \in \Sigma} F\left(\mathbf{1}, y_{1} y_{1}^{*} y_{2} y_{2}^{*} \ldots y_{k} y_{k}^{*}\right)$ as the state space of $k$ generators, and to the summand $F(\mathbf{1}, \mathbf{1} \mathbf{1} \ldots \mathbf{1} \mathbf{1})$ of $V(k)$ as the trivial summand of this state space. 


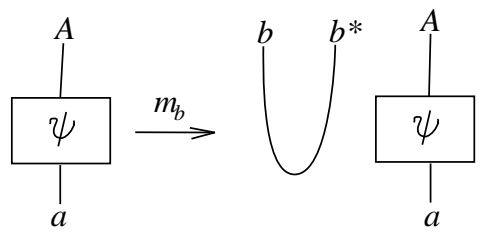

(a)

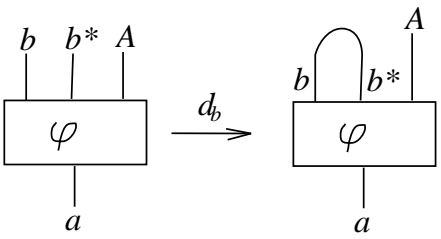

(b)

Figure 21. The morphisms $m_{b}$ and $d_{b}$.

(i) Beginning presentation. This morphism maps $F(\mathbf{1}, \mathbf{1})$ into $V(n)$ by embedding $F(\mathbf{1}, \mathbf{1})$ into the trivial summand.

$$
\begin{aligned}
& K=F(\mathbf{1}, \mathbf{1}) \rightarrow \oplus_{y_{j} \in \Sigma} F\left(\mathbf{1}, y_{1} y_{1}^{*} y_{2} y_{2}^{*} \ldots y_{n} y_{n}^{*}\right), \\
& 1 \rightarrow \quad 1 \in F(\mathbf{1}, \mathbf{1} \mathbf{1} \ldots \mathbf{1} \mathbf{1}) \text {. }
\end{aligned}
$$

(ii) Beginning relation. Starting a relation leads to the appearance of an additional generator, and therefore maps $V(n)$ into $V(n+1)$ by using the map $m_{b}$ :

$$
\oplus_{y_{j} \in \Sigma} F\left(\mathbf{1}, y_{1} y_{1}^{*} y_{2} y_{2}^{*} \ldots y_{n} y_{n}^{*}\right) \stackrel{\oplus_{b} r(b)^{2}}{\longrightarrow} m_{b^{*}} \oplus_{b, y_{j} \in \Sigma} F\left(\mathbf{1}, b^{*} b y_{1} y_{1}^{*} y_{2} y_{2}^{*} \ldots y_{n} y_{n}^{*}\right) .
$$

(iii) The morphism corresponding to a factor $\left(y_{i}\right)^{s}$ in a relation. The main ingredient in it is the circulator which is the following map of $\oplus_{x \in \Sigma} F\left(a, b x x^{*}\right)$ in itself.

$$
\begin{aligned}
C R(a, b): & \oplus_{x \in \Sigma} F\left(a, b x x^{*}\right) \stackrel{(1,3,2)}{\longrightarrow} \oplus_{x \in \Sigma} F\left(a, x\left(x^{*} b\right)\right) \stackrel{\nabla^{-1}}{\rightarrow} \\
& \oplus_{x, z \in \Sigma} F\left(a, x z^{*}\right) \otimes F\left(z^{*}, x^{*} b\right) \stackrel{i d \diamond c y c l}{\longrightarrow} \oplus_{x, z \in \Sigma} F\left(a, x z^{*}\right) \otimes F(x, b z) \stackrel{\nabla}{\rightarrow} \\
& \oplus_{z \in \Sigma} F\left(a, b z z^{*}\right) .
\end{aligned}
$$

Here $\nabla$ is as in 2.6. The diagram describing $C R(a, b)$ is presented in figure 22. We note that the circulator is actually an isomorphism and a specific expression for its inverse will be provided later. Then a factor $\left(y_{i}\right)^{s}$ in a

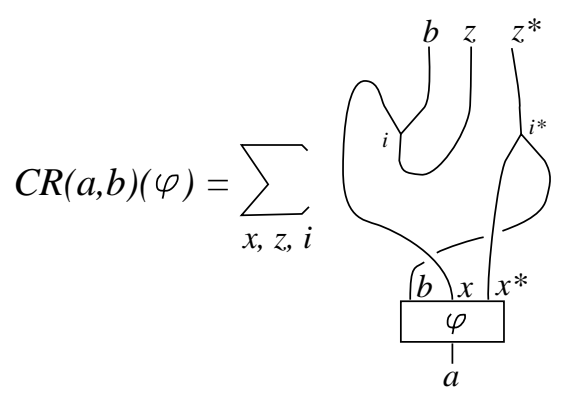

Figure 22. The circulator.

relation corresponds to the following composition of morphisms. First, in the state space of the $n+1$ generators, $y_{i} y_{i}^{*}$ is moved next to $b$. Then the part corresponding to $b y_{i} y_{i}^{*}$ is separated from the rest by using the isomorphism 
$\nabla^{-1}$, and the circulator $C R$ is applied $s$ times on it. Then following the inverse steps one goes back to the original form of the state space.

$$
\begin{aligned}
& \oplus_{b, y_{j} \in \Sigma} F\left(\mathbf{1}, b^{*} b y_{1} y_{1}^{*} y_{2} y_{2}^{*} \ldots y_{n} y_{n}^{*}\right) \rightarrow \\
& \oplus_{b, y_{j} \in \Sigma} F\left(\mathbf{1}, b^{*}\left(b y_{i} y_{i}^{*}\right) y_{1} y_{1}^{*} \ldots \hat{y}_{i} \hat{y}^{*}{ }_{i} \ldots y_{n} y_{n}^{*}\right) \stackrel{\nabla^{-1}}{\rightarrow} \\
& \oplus_{y_{j} \in \Sigma, j \neq i} \oplus_{a, b \in \Sigma} F\left(\mathbf{1}, b^{*} a y_{1} y_{1}^{*} \ldots \hat{y}_{i} \hat{y}^{*}{ }_{i} \ldots y_{n} y_{n}^{*}\right) \otimes\left(\oplus_{y_{i} \in \Sigma} F\left(a, b y_{i} y_{i}^{*}\right)\right) \stackrel{i d \otimes(C R(b, a))^{s}}{\longrightarrow} \\
& \oplus_{y_{j} \in \Sigma, j \neq i} \oplus_{a, b \in \Sigma} F\left(\mathbf{1}, b^{*} a y_{1} y_{1}^{*} \ldots \hat{y}_{i} \hat{y}^{*}{ }_{i} \ldots y_{n} y_{n}^{*}\right) \otimes\left(\oplus_{y_{i} \in \Sigma} F\left(a, b y_{i} y_{i}^{*}\right)\right) \stackrel{\nabla}{\rightarrow} \\
& \oplus_{b, y_{j} \in \Sigma} F\left(\mathbf{1}, b^{*}\left(b y_{i} y_{i}^{*}\right) y_{1} y_{1}^{*} \ldots \hat{y}_{i} \hat{y}^{*}{ }_{i} \ldots y_{n} y_{n}^{*}\right) \rightarrow \oplus_{b, y_{j} \in \Sigma} F\left(\mathbf{1}, b^{*} b y_{1} y_{1}^{*} y_{2} y_{2}^{*} \ldots y_{n} y_{n}^{*}\right),
\end{aligned}
$$

where $\hat{y}$ indicates that the corresponding term is missing.

(iv) Ending relation. This is a map from $V(n+1)$ generators back into $V(n)$ proportional to the one induced by $d_{b}$.

$$
\oplus_{b, y_{j} \in \Sigma} F\left(\mathbf{1}, b^{*} b y_{1} y_{1}^{*} y_{2} y_{2}^{*} \ldots y_{n} y_{n}^{*}\right) \stackrel{\oplus_{b} r(b)^{-1} d_{b}}{\longrightarrow} \oplus_{y_{j} \in \Sigma} F\left(\mathbf{1}, y_{1} y_{1}^{*} y_{2} y_{2}^{*} \ldots y_{n} y_{n}^{*}\right) \text {. }
$$

(v) Ending presentation. This is a map from $V(n)$ generators back to the ring $V(0)$, which is injective on the trivial summand on the state space and sends all other summands of $V(n)$ to 0 .

$$
\oplus_{y_{j} \in \Sigma} F\left(\mathbf{1}, y_{1} y_{1}^{*} y_{2} y_{2}^{*} \ldots y_{n} y_{n}^{*}\right) \rightarrow F(\mathbf{1}, \mathbf{1}), 1 \in F(\mathbf{1}, \mathbf{1} \mathbf{1} \ldots \mathbf{1} \mathbf{1}) \rightarrow 1 \text {. }
$$

THEOREM 7.3. Given a group presentation $P$, the invariant of $P$, produced by the above algorithm, is exactly $Q(P)$.

The key is to understand the powers of the circulator. Given $b \in \Sigma$ and a nonzero integer $l$, we use the definitions of $b^{l}$ and $f(b, l)$ made in 5.1. Let $x, w \in \Sigma$, $\left\{\epsilon_{i}\right\}_{i}$ be a basis for $F\left(w^{*}, x^{*} b^{l}\right)$ and let $\left\{\epsilon_{i}^{*}\right\}_{i}$ be its dual. We define morphisms $\phi(l, b, x, w, i): b x x^{*} \rightarrow x^{*}\left(b b^{l}\right) w$ and $\psi(l, b, x, w, i): x^{*}\left(b^{l} b\right) w \rightarrow b w w^{*}$ in the following way:

$$
\begin{aligned}
\phi(l, b, x, w, i): & b x x^{*} \rightarrow b x x^{*} \mathbf{1} \stackrel{(1,2,3) \diamond \Lambda_{w}}{\longrightarrow}\left(x^{*} b x\right)\left(w^{*} w\right) \stackrel{i d \diamond\left(\epsilon_{i} \diamond i d_{w}\right)}{\longrightarrow} \\
& \rightarrow\left(x^{*} b x\right)\left(x^{*} b^{l} w\right) \rightarrow x^{*}\left(b\left(x x^{*}\right) b^{l}\right) w \stackrel{\lambda_{x}}{\longrightarrow} x^{*}\left(b b^{l}\right) w, \\
\psi(l, b, x, w, i): & x^{*}\left(b^{l} b\right) w \rightarrow\left(x^{*} b^{l}\right) b w \stackrel{\epsilon_{i}^{*}}{\longrightarrow} w^{*} b w \stackrel{(1,3,2)}{\longrightarrow} b w w^{*} .
\end{aligned}
$$

Proposition 7.4. Given $f \in F\left(a, b x x^{*}\right), C R(b, a)^{l}(f)=\oplus_{w \in \Sigma} c r(l, b, x, w) \circ f$, where $\operatorname{cr}(l, b, x, w)=\sum_{i} \psi(l, b, x, w, i) \circ\left(i d_{x^{*}} \diamond f(b, l) \diamond i d_{w}\right) \circ \phi(l, b, x, w, i)$.

The corresponding diagram is shown in figure 23. Note that $\operatorname{cr}(l, b, x, w)$ can actually be written as the composition of a partial trace and another morphism, and therefore it is independent on the particular choice of the basis $\left\{\epsilon_{i}\right\}_{i}$. The fact that the circulator $C R(a, b): F\left(a, b x x^{*}\right) \rightarrow F\left(a, b w w^{*}\right)$ acts as $\operatorname{cr}(1, b, x, w)$ follows from the comparison of the diagrams in figure 22 and 23 (a). Then in figure 24 we prove that $\operatorname{cr}(-1, b, x, w)$ is a right inverse of the circulator. In a similar fashion one can see that it is a left inverse as well. Then, by assuming that for $l>1$, the $(l-1)$-th power of the circulator acts as $\operatorname{cr}(l-1, b, x, w)$, figure 25 proves that the statement is also true for the $l$-th power. The inductive proof for the negative powers of the circulator goes in a similar fashion.

Now theorem 7.3 follows from the following statement. Let $P$ be a presentation with $n$ generators, $R=x_{i_{1}}^{l_{1}} x_{i_{2}}^{l_{2}} \ldots x_{i_{s}}^{l_{s}}$ be a relation in $P$, and given $\underline{y}, \underline{z} \in \Sigma^{\times n}$ and $b \in \Sigma$, let $\left\{\epsilon_{j}^{i}\left(z_{i}^{*}, y_{i}^{*} g_{i}(R, b)\right)\right\}_{j}, i=1 \div n$, be a set of bases for the homomorphisms 




(a) $c(|l|, b, x, w)$



(b) $c(-|l|, b, x, w)$

Figure 23. The morphism $c(l, b, x, w)$.

spaces $F\left(z_{i}^{*}, y_{i}^{*} g_{i}(R, b)\right)$. Then the map $V(n+1) \rightarrow V(n+1)$, which the relation $R$ induces according to the algorithm in 7.2 , is given by $\varphi \rightarrow \oplus_{\underline{y}, \underline{z}, j} r l(\underline{y}, \underline{z}, j) \circ \varphi$, where $r l(\underline{y}, \underline{z}, j)$ is the following composition of maps:

$$
\begin{aligned}
& b^{*} b y_{1} y_{1}^{*} y_{2} y_{2}^{*} \ldots y_{n} y_{n}^{*} \rightarrow b^{*}\left(\diamond_{i} y_{i}^{*}\right) b\left(\diamond_{i} y_{i}\right) \stackrel{i d \diamond\left(\diamond_{i} \Lambda_{z_{i}}\right)}{\longrightarrow} \\
& b^{*}\left(\diamond_{i} y_{i}^{*}\right) b\left(\diamond_{i} y_{i}\right)\left(\diamond_{i} z_{i}^{*} z_{i}\right) \stackrel{i d \diamond\left(\diamond_{i}\left(\epsilon_{j}^{\diamond_{j}} \diamond_{z_{i}}\right)\right)}{\longrightarrow} \\
& b^{*}\left(\diamond_{i} y_{i}^{*}\right) b\left(\diamond_{i} y_{i}\right)\left(\diamond_{i} y_{i}^{*} g_{i}(R, b) z_{i}\right) \rightarrow b^{*}\left(\diamond_{i} y_{i}^{*}\right) b\left(\diamond_{i} y_{i} y_{i}^{*}\right)\left(\diamond_{i} g_{i}(R, b)\right)\left(\diamond_{i} z_{i}\right) \stackrel{i d \diamond\left(\diamond_{i} \lambda_{y_{i}}\right) \diamond i d}{\longrightarrow} \\
& b^{*}\left(\diamond_{i} y_{i}^{*}\right) b\left(\diamond_{i} g_{i}(R, b)\right)\left(\diamond_{i} z_{i}\right) \stackrel{i d \diamond \phi(R) \diamond i d}{\longrightarrow} b^{*}\left(\diamond_{i} y_{i}^{*}\right)\left(\diamond_{i} g_{i}(R, b)\right) b\left(\diamond_{i} z_{i}\right) \rightarrow \\
& b^{*}\left(\diamond_{i} y_{i}^{*} g_{i}(R, b)\right) b\left(\diamond_{i} z_{i}\right) \stackrel{i d \diamond\left(\diamond_{i} \epsilon_{j}^{i *}\right) \diamond i d}{\longrightarrow} b^{*}\left(\diamond_{i} z_{i}^{*}\right) b\left(\diamond_{i} z_{i}\right) \rightarrow b^{*} b z_{1} z_{1}^{*} z_{2} z_{2}^{*} \ldots z_{n} z_{n}^{*},
\end{aligned}
$$

where $\phi(R)=\left(i d_{b} \diamond \kappa(R)\right)[R, b]^{o}\left(\kappa(R)^{-1} \diamond i d_{b}\right): b\left(\diamond_{i} g_{i}(R, b)\right) \rightarrow\left(\diamond_{i} g_{i}(R, b)\right) b$ (see 5.4. The corresponding diagram is presented in figure 26, where for simplicity we have restricted ourselves to the case of two generators. The statement is proved by induction over $s$. For $s=1$ it is reduced to proposition 7.4. Assume it is true for $R$, and let $R^{\prime}=R x_{k}^{l}$ for some $k$ and $l$. Then

$$
g_{i}\left(R^{\prime}, b\right)=\left\{\begin{array}{l}
g_{k}(R, b) b^{l} \text { if } i=k, \\
g_{i}(R, b) \text { otherwise }
\end{array}, \quad \text { and } \quad\left[R^{\prime}, b\right]=\circ_{b}\left([R, b]^{o} \circ_{b} f(b, l)\right) .\right.
$$

Then the statement follows from figure 27.

\section{Conjectures and consequences}

As we said, this work was inspired by an extensive numerical study of the invariants defined in [8]. The numerical project has been carried out by Frank Quinn, the author, and Luoqi Zhang, and the autonomous tensor categories used in it are subcategories of the Gelfand-Kazhdan categories, as described in 2.7. We remind that such a subcategory is determined by a type and rank of simple Lie algebra $(A, B, C, D, E$ or $F)$, a prime number $p$, and an invariant sublattice of the weight 

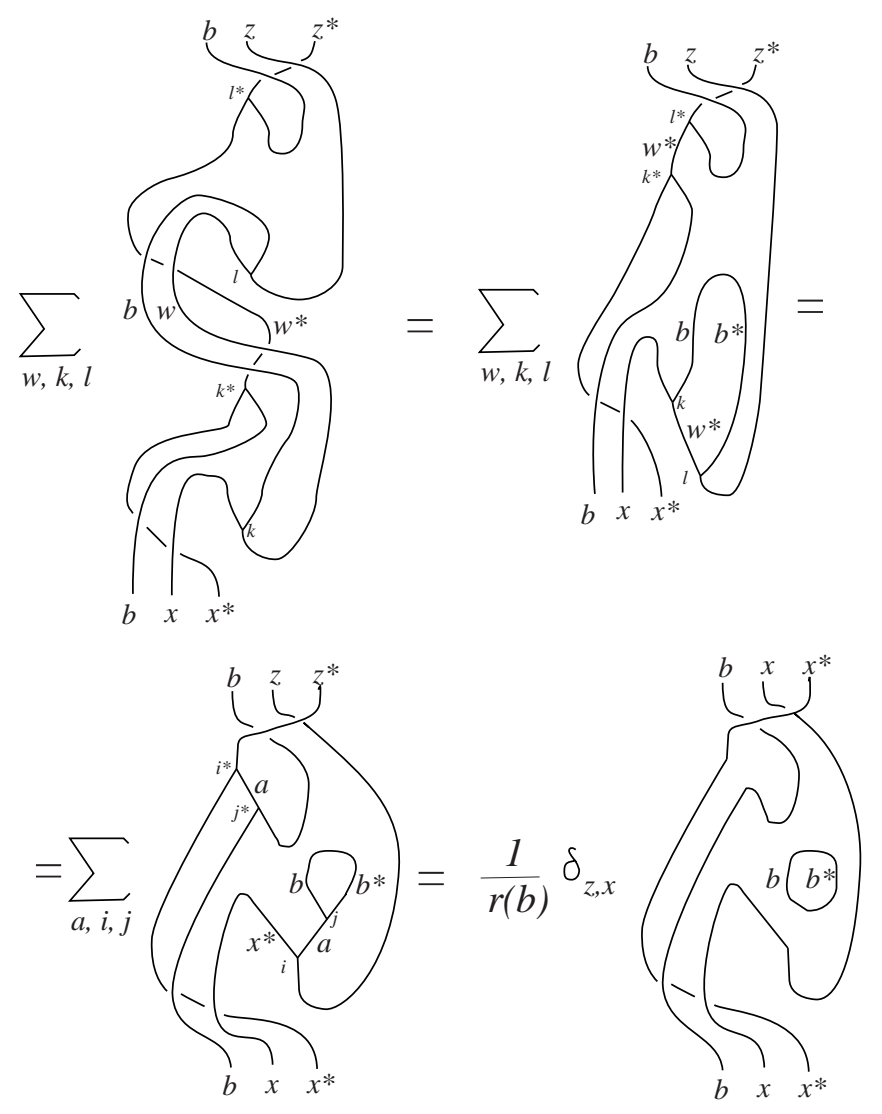

FiguRE 24. Proof that $c(-1, b, x, w)$ represents the inverse of $c(1, b, x, w)$.

lattice (containing the root lattice). But actually many of the categories corresponding to different algebras are equivalent. Conjecturally, any two autonomous tensor categories with sets of simple objects $\Sigma_{1}$ and $\Sigma_{2}$, such that there is a bijection $\mu: \Sigma_{1} \rightarrow \Sigma_{2}$, and $\operatorname{dim}(F(a, b c))=\operatorname{dim}(F(\mu(a), \mu(b) \mu(c)))$, are equivalent. In other words, the dimensions of the homomorphism spaces determine the category. Let $\operatorname{dim}(a, b c)$ denote the dimension of $F(a, b c)$ and we will refer to those as dimension functions. On web page http://www.math.vt.edu/quantum_topology has been collected a list with the values of the dimension functions for some categories corresponding to algebras type $A, B, C, D$, small primes, and either the full weight lattice or the root lattice. Let $L$ be the order of the abelian group obtained as a quotient of the lattice over which the category is defined, modulo the root lattice. Then all numerically generated examples satisfy the following conjecture:

Conjecture 8.1. In the case of stable Gelfand-Kazhdan categories, for any $b \in \Sigma$

(a) $\operatorname{dim}\left(a, b^{L p}\right)=0 \quad(\bmod p)$, if $a \neq \mathbf{1}$;

(b) $r(b)^{-1} \sum_{c \in \Sigma} \operatorname{dim}\left(b, c c^{*}\right)=\left\{\begin{array}{c}|\Sigma| \text { if } b \text { belongs to the root lattice, } \\ 0 \text { otherwise }\end{array} \quad(\bmod p)\right.$. 


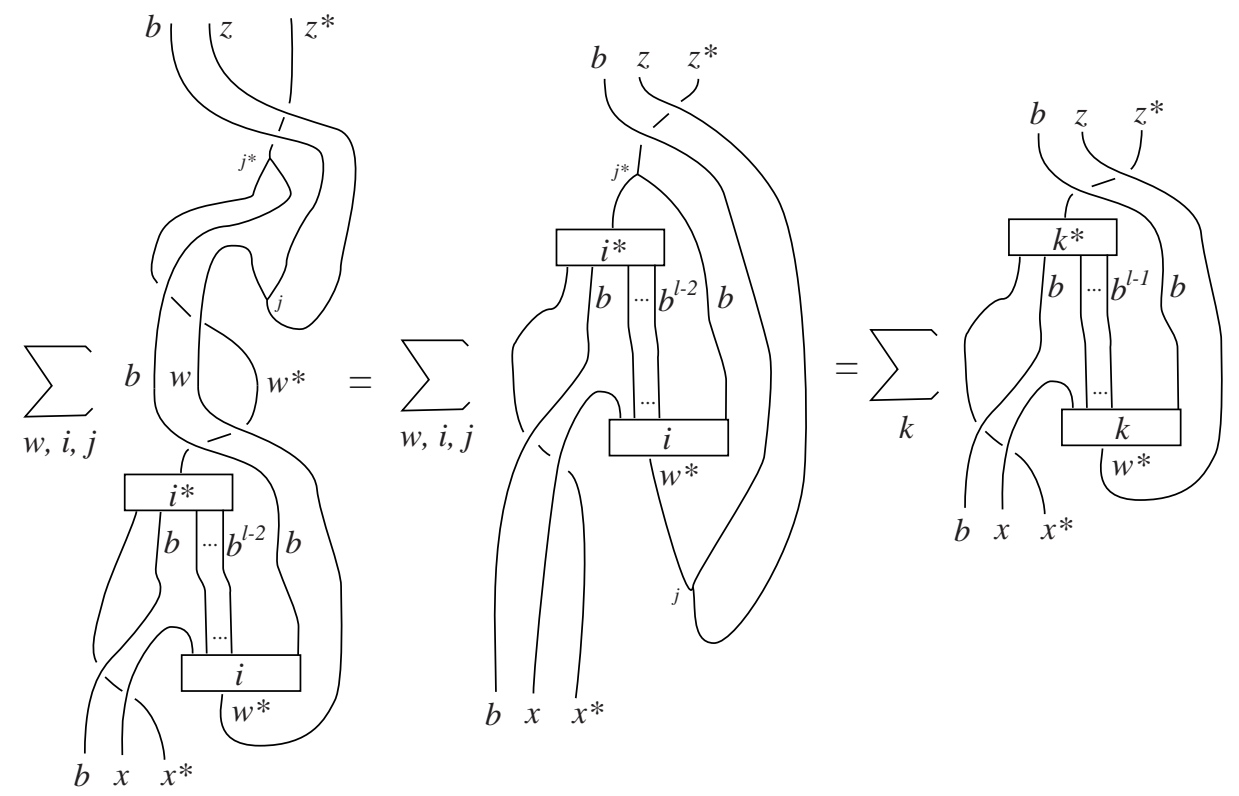

FIgURE 25. Inductive step in the proof of proposition 7.4 .

We make few comments on this. From 3.8 and (a) above we obtain

$$
\operatorname{dim}\left(\mathbf{1}, b^{L p}\right)=\sum_{a \in \Sigma} \operatorname{dim}\left(a, b^{L p}\right) r(a)=r\left(b^{L p}\right)=r(b)^{L p}=r(b)^{L}, \quad(\bmod p) .
$$

In connection with (b) we comment that the statement can be proved easily for algebra type $A_{1}$. Moreover, the 0 part can be derived from a conjecture of Frank Quinn, according to which the category over an arbitrary lattice is a product of the category over the root lattice and a finite group category. If this is true, it would imply that the only interesting categories are the ones coming from the root lattice, since a finite group category brings to a classical invariant. For categories over the root lattice, the conjecture 8.1 brings to the following result.

Corolary 8.2. Let $P=<x_{1}, x_{2}, \ldots, x_{n} \mid R_{1}, R_{2}, \ldots, R_{m}>$ be a group presentation. If we are working with a Gelfand-Kazhdan category over the root lattice, and 8.1 (b) is true, then $Q\left(P^{\prime}\right)=|\Sigma| Q\left(P^{\prime \prime}\right)$, where

$$
\begin{aligned}
& P^{\prime}=<x_{1}, x_{2}, \ldots, x_{n}, y \mid R_{1}, R_{2}, \ldots, R_{m}, x_{k} y x_{k}^{-1} y^{-1}>\text { and } \\
& P^{\prime \prime}=<x_{1}, x_{2}, \ldots, x_{n} \mid R_{1}, R_{2}, \ldots, R_{m}, x_{k}>.
\end{aligned}
$$

Let $\underline{b} \in \Sigma^{\times m}$, and $a, c \in \Sigma$. If $(\underline{b}, c)$ denotes the sequence $\left(b_{1}, b_{2}, \ldots, b_{m}, c\right)$ we have that

$$
\begin{gathered}
{\left[P^{\prime},(\underline{b}, c)\right]=[P, \underline{b}] \diamond\left[x_{k} y x_{k}^{-1} y^{-1}, c\right]: \operatorname{Rel}(\underline{b}) c c c^{*} c^{*} \rightarrow \operatorname{Rel}(\underline{b}) c c c^{*} c^{*},} \\
G_{l}^{\prime}(\underline{b}, c)=\left\{\begin{array}{l}
G_{k}(\underline{b})\left(c c^{*}\right) \text { if } l=k, \quad \text { and } \quad G_{y}^{\prime}(\underline{b}, c)=c c^{*}, \\
G_{l}(\underline{b}) \text { otherwise, }
\end{array}\right. \\
\xi\left(P^{\prime}\right): \operatorname{Rel}(\underline{b}) c c c^{*} c^{*} \stackrel{\xi(P) \diamond(2,3)}{\longrightarrow}\left(\diamond_{l} G_{l}(\underline{b})\right) c c^{*} c c^{*} \rightarrow \\
\rightarrow\left(\diamond_{l<k} G_{l}(\underline{b})\right) G_{k}(\underline{b})\left(c c^{*}\right)\left(\diamond_{l>k} G_{l}(\underline{b})\right) c c^{*} .
\end{gathered}
$$




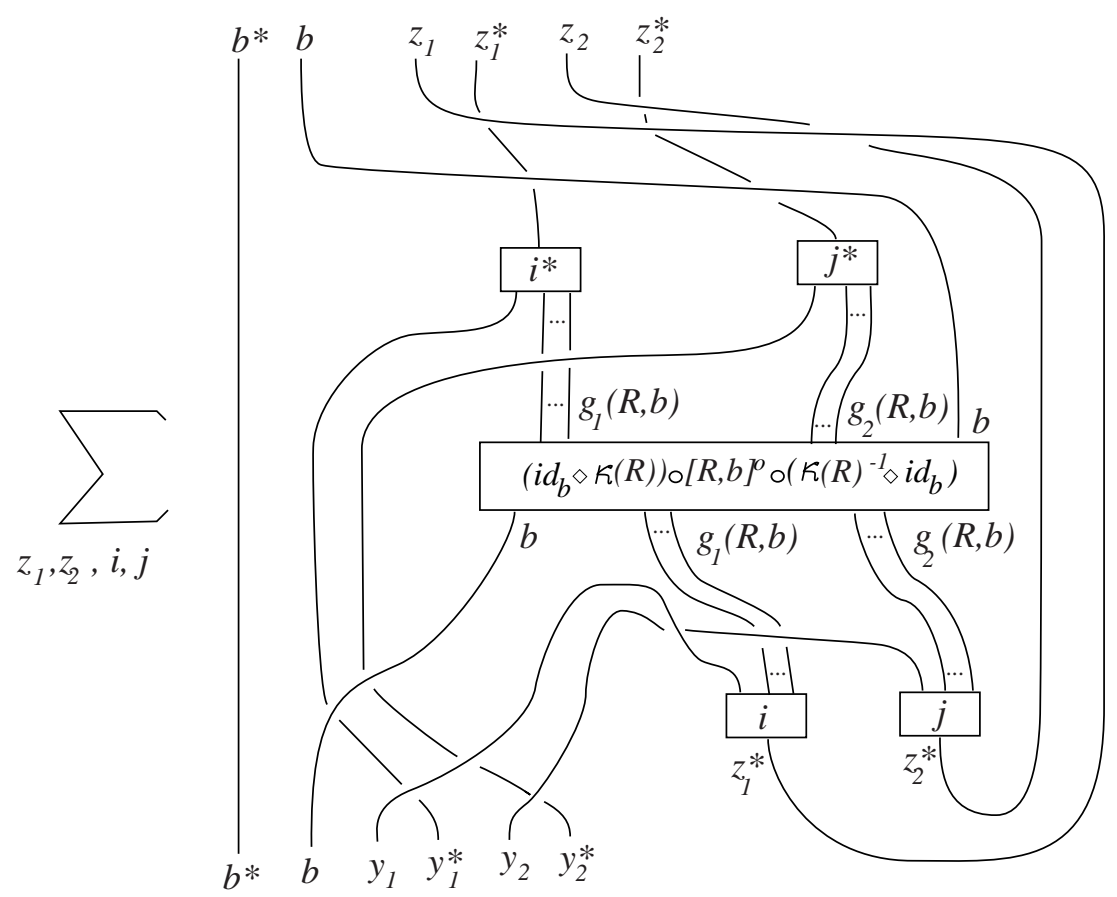

Figure 26. The morphisms $V(n+1) \rightarrow V(n+1)$ induced by a relation $R$ (case $n=2$ ).

Moreover,

$$
\begin{aligned}
& {\left[P^{\prime \prime},(\underline{b}, a)\right]=r(a)[P, \underline{b}] \diamond i d_{a}: \operatorname{Rel}(\underline{b}) a \rightarrow \operatorname{Rel}(\underline{b}) a,} \\
& G_{l}^{\prime \prime}(\underline{b}, a)=\left\{\begin{array}{l}
G_{k}(\underline{b}) a \text { if } l=k, \\
G_{l}(\underline{b}) \text { otherwise },
\end{array}\right. \\
& \xi\left(P^{\prime \prime}\right)=\operatorname{Rel}(\underline{b}) a \stackrel{\xi(P) \diamond i d_{a}}{\longrightarrow}\left(\diamond_{l} G_{l}(\underline{b})\right) a \rightarrow\left(\diamond_{l<k} G_{l}(\underline{b})\right) G_{k}(\underline{b}) a\left(\diamond_{l>k} G_{l}(\underline{b})\right),
\end{aligned}
$$

where we have used the notations in 5.5. Then by using 4.2 (c) and (d) we obtain the following for the part of the partial trace involving $G_{k}^{\prime}(\underline{b}, c)$ and $G_{y}^{\prime}(\underline{b}, c)$ :

$$
\begin{aligned}
W & =\sum_{c \in \Sigma} \operatorname{Tr}_{\left\{G_{k}^{\prime}(\underline{b}, c) \rightarrow \mathbf{1}, G_{y}^{\prime}(\underline{b}, c) \rightarrow \mathbf{1}\right\}}\left(\xi\left(P^{\prime}\right) \circ\left([P, \underline{b}] \diamond\left[x_{k} y x_{k}^{-1} y^{-1}, c\right]\right) \circ \xi\left(P^{\prime}\right)^{-1}\right)= \\
& =\sum_{c, a \in \Sigma} \operatorname{Tr}_{G_{k}(\underline{b}) a \rightarrow \mathbf{1}}\left(\xi\left(P^{\prime \prime}\right) \circ([P, \underline{b}] \diamond K) \circ \xi\left(P^{\prime \prime}\right)^{-1}\right),
\end{aligned}
$$

where $K=\operatorname{Tr}_{\left\{c c^{*} \rightarrow a, c c^{*} \rightarrow \mathbf{1}\right\}}\left((2,3) \circ\left[x_{k} y x_{k}^{-1} y^{-1}, c\right] \circ(2,3)\right)$. Figure 28 shows that in fact, $K=\operatorname{dim}\left(a, c c^{*}\right) i d_{a} \diamond i d_{\mathbf{1}}$. Hence we obtain :

$$
\begin{aligned}
W & =\sum_{c, a \in \Sigma} \operatorname{dim}\left(a, c c^{*}\right) \operatorname{Tr}_{G_{k}(\underline{b}) a \rightarrow \mathbf{1}}\left(\xi\left(P^{\prime \prime}\right) \circ\left([P, \underline{b}] \diamond i d_{a}\right) \circ \xi\left(P^{\prime \prime}\right)^{-1}\right)= \\
& =|\Sigma| \sum_{a \in \Sigma} \operatorname{Tr}_{G_{k}(\underline{b}) a \rightarrow \mathbf{1}}\left(\xi\left(P^{\prime \prime}\right) \circ\left[P^{\prime \prime},(\underline{b}, a)\right] \circ \xi\left(P^{\prime \prime}\right)^{-1}\right),
\end{aligned}
$$

which is exactly the part of the partial trace in the definition of $Q\left(P^{\prime \prime}\right)$ involving $G_{k}^{\prime \prime}(\underline{b}, a)$. 


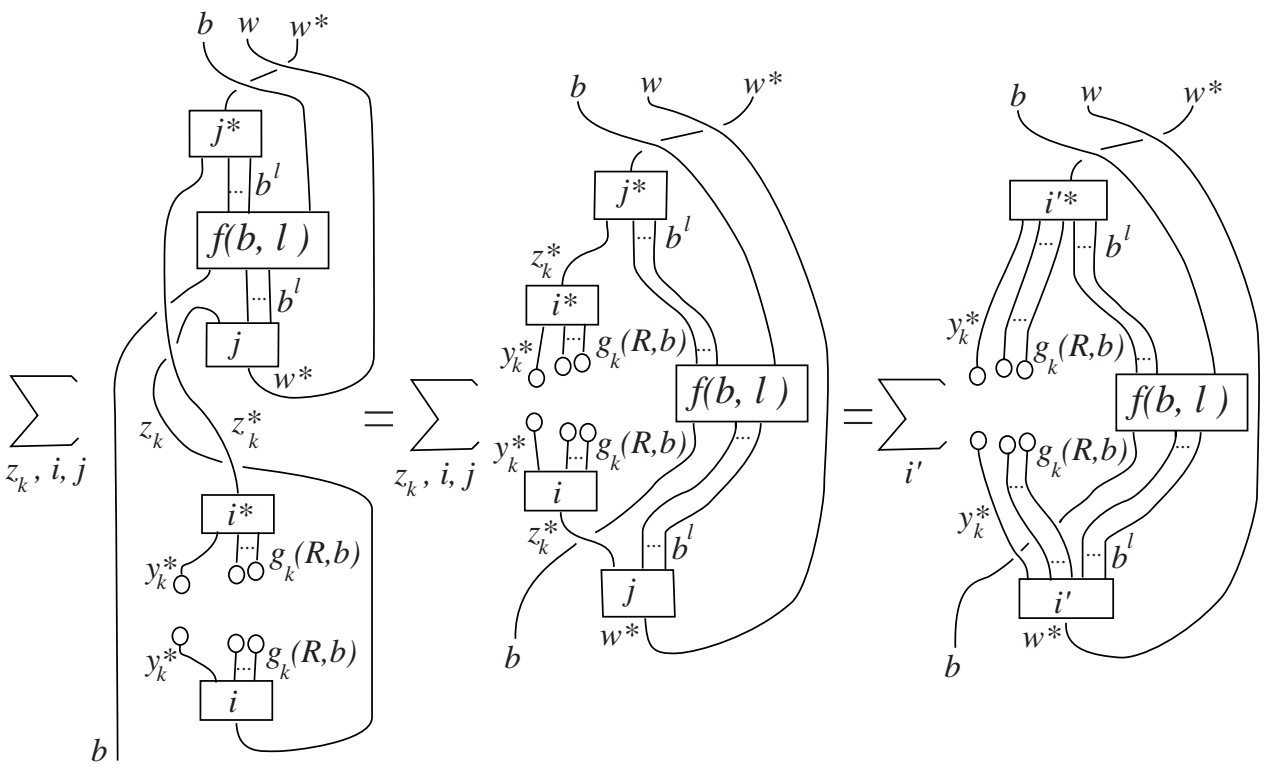

Figure 27. Inductive step in the proof of theorem 7.3 .

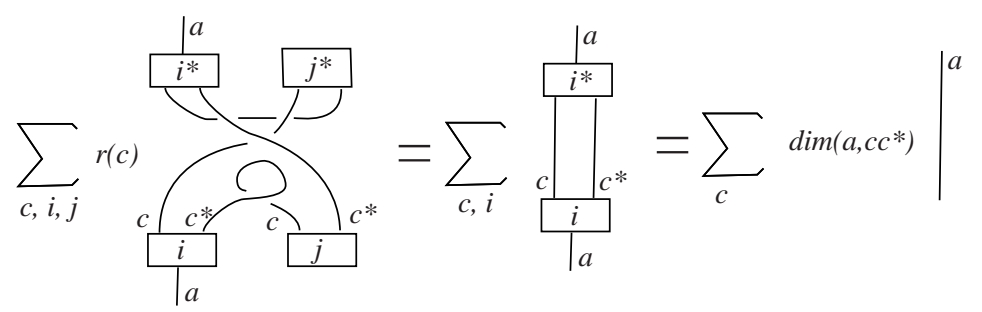

Figure 28. Proof of corollary 8.2 .

Another conjecture we would like to discuss concerns the order of the circulator. It is observed in [8], that for all numerically generated categories, the order of the circulator is in fact $L p$. On the basis of this and the expression for the powers of the circulator 7.4 we may state the following

Conjecture 8.3. Let $\sigma^{L p+1}(b)=(1,2,3, \ldots, L p+1): b b^{L p} \rightarrow b b^{L p}$. Then in the case of stable Gelfand-Kazhdan categories,

$$
\operatorname{Tr}_{b^{L p} \rightarrow w} \sigma^{L p+1}(b)= \begin{cases}0 & \text { if } w \neq \mathbf{1} \\ i d_{b} \diamond i d_{1} & \text { otherwise }\end{cases}
$$

The corresponding diagram is presented in figure 29.

The conjecture about the finite order of the circulator implies that given a presentation, the exponents of the generators in the relations matter only mod $L p$, in particular it is enough to look at presentations $P$ with only positive exponents. In this last case, the morphism $[P, \underline{b}]$ has a particularly simple form - it is a product 


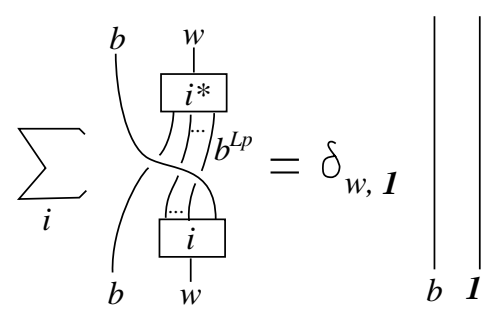

Figure 29. Conjecture 8.3 .

of cyclic permutations:

$$
[P, \underline{b}]=\left(1,2,3, \ldots, e_{1}\right) \diamond\left(1,2,3, \ldots, e_{2}\right) \diamond \cdots \diamond\left(1,2,3, \ldots, e_{m}\right),
$$

where $e_{i}$ is the total exponent of the relation $R_{i}$. This fact doesn't seem to help though with evaluating the invariant, at least until some more information is available for the symmetry group representations $\rho\left[b^{k}\right]$.

In conclusion we point out that clearly the main question is how the invariant behaves under the move (vii) in section 1, i.e. under simple homotopy equivalence. The numerical examples haven't exhibited any nontrivial behavior, but using the formalism in this paper to write down the change in the value of the invariant under (vii), brings to expressions which look quite complicated and we don't know how to analyze. We suspect that if the behavior of the computed invariant under this move is actually trivial, this is a consequence of some specific properties of the Gelfand Kazhdan categories being used ( like the ones listed in conjecture 8.1), rather then a consequence of the general setup of the theory.

\section{References}

[1] J.Andrews, M.Curtis, Free groups and handlebodies, Proc.AMS 16 (1965),192-195.

[2] I.Bobtcheva, Numerical generation of semisimple tortile categories coming from quantum groups, PhD thesis, Virginia Polytechnic Institute and State University, Blacksburg, VA, August 1996.

[3] P.Freyd and D.Yetter, Brided compact closed categories with applications in law-dimensional topology, Adv. in Math. 77 (1989),156-182.

[4] S.Gelfand and D.Kazhdan, Examples of tensor categories, Invent.Math. 109 (1992),595-617.

[5] C.Hog-Angeloni, W.Metzler and A.Sieradski, Two-dimensional homotopy and combinatorial group theory, Cambridge University Press, London Mathematical Society Lecture Notes Series, vol.197, 1993.

[6] G.Kelly and M.Laplaza, Coherence for compact closed categories, J.Pure and Applied Math. 19 (1980), 193-213.

[7] S.MacLane, Natural associativities and commutativities, Rice Univ. Studies 49 (1963),28-46.

[8] F.Quinn, Lectures on Axiomatic Topological Quantum Field Theory, LAS/Park City Mathematical Series, vol.1, 1995.

[9] I.Bobtcheva and F.Quinn, Numerical presentations of tortile categories, internal report.

[10] F.Quinn, Handlebodies and 2-complexes, Lecture Notes in Mathematics 1167, Geometry and Topology, Proceedings, University of Maryland, 1983-1984, ,245-259.

[11] Mei Chee Shum, Tortile tensor categories, Journal of Pure and Applied Algebra 93 Berlin Heidelberg New York (1994), 57-110.

[12] D.Yetter, State-sum invariants of 3-manifolds associated to artinian semisimple tortile categories, Topology and its Applications 58 (1994),47-80.

Virginia Polytechnic Institute and State University Blacksburg, VA, 24061

E-mail address: demeio@anvax1.cineca.it 